\title{
NAD+ improves neuromuscular development in a zebrafish model of FKRP- associated dystroglycanopathy
}

\author{
Erin C. Bailey ${ }^{1,4}$, Sarah S. Alrowaished ${ }^{1}$, Elisabeth A. Kilroy ${ }^{4}$, Emma S. Crooks ${ }^{1}$, Daisy M. Drinkert ${ }^{3}$,
} Chaya M. Karunasiri ${ }^{1,5}$, Joseph J. Belanger ${ }^{1,6}$, Andre Khalii ${ }^{2,4}$, Joshua B. Kelley ${ }^{3,4}$ and Clarissa A. Henry ${ }^{1,4^{*}}$ (D)

\begin{abstract}
Background: Secondary dystroglycanopathies are muscular dystrophies that result from mutations in genes that participate in Dystroglycan glycosylation. Glycosylation of Dystroglycan is essential for muscle fibers to adhere to the muscle extracellular matrix (myomatrix). Although the myomatrix is disrupted in a number of secondary dystroglycanopathies, it is unknown whether improving the myomatrix is beneficial for these conditions. We previously determined that either NAD+ supplementation or overexpression of Paxillin are sufficient to improve muscle structure and the myomatrix in a zebrafish model of primary dystroglycanopathy. Here, we investigate how these modulations affect neuromuscular phenotypes in zebrafish fukutin-related protein ( $f k r p)$ morphants modeling FKRP-associated secondary dystroglycanopathy.
\end{abstract}

Results: We found that NAD+ supplementation prior to muscle development improved muscle structure, myotendinous junction structure, and muscle function in fkrp morphants. However, Paxillin overexpression did not improve any of these parameters in fkrp morphants. As movement also requires neuromuscular junction formation, we examined early neuromuscular junction development in fkrp morphants. The length of neuromuscular junctions was disrupted in fkrp morphants. NAD+ supplementation prior to neuromuscular junction development improved length. We investigated NMJ formation in dystroglycan (dag1) morphants and found that although NMJ morphology is disrupted in dag1 morphants, NAD+ is not sufficient to improve NMJ morphology in dag 1 morphants. Ubiquitous overexpression of Fkrp rescued the fkrp morphant phenotype but muscle-specific overexpression only improved myotendinous junction structure.

Conclusions: These data indicate that Fkrp plays an early and essential role in muscle, myotendinous junction, and neuromuscular junction development. These data also indicate that, at least in the zebrafish model, FKRP-associated dystroglycanopathy does not exactly phenocopy DG-deficiency. Paxillin overexpression improves muscle structure in dag1 morphants but not fkrp morphants. In contrast, NAD+ supplementation improves NMJ morphology in fkrp morphants but not dag1 morphants. Finally, these data show that muscle-specific expression of Fkrp is insufficient to rescue muscle development and homeostasis.

Keywords: Zebrafish, Dystroglycanopathy, FKRP, NAD+, Neuromuscular junction

\footnotetext{
* Correspondence: Clarissa.Henry@maine.edu

${ }^{1}$ School of Biology and Ecology, University of Maine, Orono, ME 04469, USA

${ }^{4}$ Graduate School of Biomedical Sciences and Engineering, University of

Maine, 217 Hitchner Hall, Orono, ME 04469, USA

Full list of author information is available at the end of the article
}

(c) The Author(s). 2019 Open Access This article is distributed under the terms of the Creative Commons Attribution 4.0 International License (http://creativecommons.org/licenses/by/4.0/), which permits unrestricted use, distribution, and reproduction in any medium, provided you give appropriate credit to the original author(s) and the source, provide a link to the Creative Commons license, and indicate if changes were made. The Creative Commons Public Domain Dedication waiver (http://creativecommons.org/publicdomain/zero/1.0/) applies to the data made available in this article, unless otherwise stated. 


\section{Background}

Muscle is a highly conserved tissue that is part of the neuromusculoskeletal system and is essential for strength, locomotion, and health. The neuromuscular junction (NMJ) initiates muscle contraction, whereas the myotendinous junction (MTJ) is the major site of force transmission from muscle to the skeletal system. Muscle fibers adhere to their surrounding extracellular matrix (ECM), the MTJ, and the NMJ; and these adhesion complexes are critical for muscle development and homeostasis (reviewed in [26]). The muscle extracellular matrix and adhesion complexes at MTJs and NMJs are specialized and allow the muscle to adhere to and interact with neurons and tendons during development and homeostasis. Thus, regulation of cell-ECM adhesion is essential for development and homeostasis of the neuromusculoskeletal system.

The dystrophin-glycoprotein complex (DGC) is an essential component of muscle-ECM adhesion. The DGC is a multi-protein complex that indirectly links the actin cytoskeleton of muscle fibers to the lamininrich basement membrane in the ECM [18]. The DGC is thought to provide mechanical stabilization during muscle contraction $[18,60]$ in addition to its role as a signaling complex [12]. Different variants of the DGC are present at the MTJ versus the NMJ. Whereas Dystrophin is the predominant protein that connects the actin cytoskeleton to the transmembrane protein Dystroglycan (DG) at the MTJ [18], Utrophin mainly establishes this connection at the NMJ [16]. Despite these differences, DG is present and highly glycosylated at both the MTJ and the NMJ. DG glycosylation is required for DG binding to basement membrane proteins such as laminin, agrin, and perlecan $[8,20$, 49]. Dystroglycanopathies result from mutations in genes responsible for glycosylation of alpha-DG (DG contains two subunits: a transmembrane beta subunit and an extracellular alpha subunit) $[23,33,51]$. Thus far, 16 gene products that participate in DG glycosylation have been identified [52]. In addition to muscle degeneration, dystroglycanopathies are frequently associated with central nervous system defects such as intellectual disability and brain malformation [52]. The role of DG glycosylation in the peripheral nervous system is less understood although abnormal NMJs have been reported after birth in a couple of dystroglycanopathy mouse models [13, 32, 43, 54, 61]. Because NMJ defects have been observed after embryonic development, the most prevalent hypothesis is that postsynaptic DG does not play a role in NMJ formation, but is important for NMJ stabilization [52]. For example, patients with mutations in GMPPB (required for GDP-mannose formation [36]) show decreased action potentials with repeated nerve stimulation [37]. Given the plethora of glycosylated proteins at the
NMJ, it is possible that a subset of dystroglycanopathy genes may be required outside of muscle tissue for NMJ development.

FKRP-associated dystroglycanopathy results from mutations in FKRP, which encodes an enzyme critical for DG glycosylation. FKRP works in concert with Fukutin to transfer CDP-ribitol synthesized by Isoprenoid Synthase Domain-Containing (ISPD) protein to alpha-DG $[21,38]$ to form a tandem ribitol5 -phosphate that links xylose with $N$-acetylgalactosamine [69]. This process is necessary for DG glycosylation, which is important for DG binding to basement membrane proteins [8, 20, 49]. Patients with FKRP-associated dystroglycanopathy present with phenotypic variability; however, muscle weakness and elevated serum creatine kinase are consistently present [37]. Depending on the molecular basis of the FKRP mutation, individuals with this condition may develop limb-girdle muscular dystrophy 2I (LGMD2I) or congenital muscular dystrophy with or without eye and brain involvement $[67,75]$. There is some genotype-phenotype correlation in patients with C-terminal FKRP mutations tending to be more severe $[4,6,67,75]$. However, there is remarkable phenotypic variation among individuals with the same mutation [67]. For example, a study of 25 patients homozygous for c826C/A found significant variation in muscle pathology, and levels of glycosylated DG [67]. Neither the histopathological alterations nor levels of DG glycosylation correlated with age of onset or walking function. Interestingly, whereas one set of siblings presented with similar clinical and histopathological features, a second set of siblings had dramatic variation in the age of onset (12 vs 27 years old) [67]. These data suggest that there are modifying factors-environmental and/or genetic-that affect progression of FKRP-associated dystroglycanopathy. These data also suggest that it is imperative to study features other than muscle pathology and/or levels of DG glycosylation. Mouse [1] and zebrafish models of FKRP deficiency have been used to study mechanisms associated with FKRP-associated dystroglycanopathy. Zebrafish models of FKRP-associated dystroglycanopathy include morpholino (MO) models $[39,72]$ and a more recently generated fkrp mutant [63]. These studies indicate that the fkrp morphants phenocopy the fkrp mutant, that fkrp deficiency results in impaired muscle development and wasting, and that laminin deposition at the MTJ is disrupted in Fkrp-deficient zebrafish.

Laminin is a major component of the basement membrane that surrounds muscle. Laminin is a heterotrimeric protein with an alpha, beta, and gamma chain. Adhesion of muscle fibers to laminin is 
necessary for muscle development and homeostasis $[2,28,29,64,65,80]$. The principle laminin isoform in mature vertebrate skeletal muscle is laminin-211 (alpha2 beta1 gamma1). Mutations in the human laminin alpha2 gene result in MDC1A, a common congenital muscular dystrophy [31]. A different laminin isoform, laminin-111, is the major isoform expressed during muscle development, and laminin111 is required for muscle development in mouse and zebrafish $[2,65]$. Laminin-111 can partially compensate for laminin-211. Overexpression of laminin alpha1 slows the progression of dystrophy in laminin alpha 2 mutant mice $[19,57]$. Injection of laminin111 protein directly into muscle of $m d x$ mice modeling Duchenne muscular dystrophy (DMD) improves muscle structure and function [56]. These data indicate that understanding mechanisms that mediate laminin-111 expression and polymerization during muscle development could provide information for future therapeutic development. We identified a novel pathway required for laminin-111 organization at the zebrafish MTJ during muscle development. Nicotinamide Riboside Kinase 2b (previously called muscle Integrin binding protein) $[44,45]$ is necessary for normal laminin-111 organization [24]. Yeast and human Nrk2s function in an alternative salvage pathway that generates Nicotinamide Adenine Dinucleotide (NAD+) [7, 70]. Exogenous NAD+ rescues MTJ morphogenesis in Nrk2b-deficient zebrafish, indicating that zebrafish Nrk2b also functions to generate NAD+ [24]. Given that NAD+ biosynthesis is necessary for normal laminin-111 organization during muscle development, we asked whether exogenous NAD+ would be sufficient to improve muscle structure and function in zebrafish modeling muscular dystrophies. NAD+ supplementation increases laminin organization and reduces muscle degeneration in zebrafish deficient for either of the laminin-211 receptors (DG or Integrin alpha7 (Itga7)) [25]. As vitamin B3 is a precursor for $\mathrm{NAD}+$, we asked whether vitamin supplementation would increase NAD+ and improve muscle structure and function in DG-deficient zebrafish. Supplementation with EmergenC packets that contain B vitamins (chosen because they are water soluble) improves muscle structure and motility in DG-deficient zebrafish.

The two major transmembrane receptors that anchor muscle cells to laminin in their ECM are DG (described above) $[18,20]$ and Integrin alpha7 (Itga7) $[11,66]$. Itga7 is also required for muscle homeostasis: mutations in Itga7 lead to congenital muscular dystrophy with Itga7 deficiency [30]. These two cell adhesion complexes display some degree of functional redundancy in both zebrafish and mouse models. Pharmacologically increasing levels of Itga7 compensates for the loss of Dystrophin in a mouse model of DMD [62, 76]. Given that NAD+ is sufficient to improve muscle structure in zebrafish deficient for either laminin receptor complex (DG or Itga7), we hypothesized that NAD+ increases laminin organization by increasing clustering of the remaining receptor [25]. Our model is that in the absence of Itga7, NAD+ increases DG clustering, thus improving muscle-ECM adhesion. Similarly, we hypothesize that in the absence of DG, NAD+ increases Itga7 clustering, thus improving muscle-ECM adhesion. This model leads to the question of whether, if DG is present but hypoglycosylated, NAD+ would be sufficient to improve muscle structure in a zebrafish secondary dystroglycanopathy model. Our previous data showed beneficial effects of NAD + when administered prior to muscle development [25]. Whether NAD+ is beneficial after initial muscle development in this context has not yet been determined.

Paxillin is an Integrin-associated adaptor protein that concentrates at the MTJ during muscle development [14]. Paxillin is an essential signaling nexus that regulates cell adhesion, morphology, and migration [15]. Paxillin participates in the Nrk2b-laminin pathway. Nrk2b is cell-autonomously required for subcellular concentration of Paxillin at the MTJ, and Paxillin overexpression rescues muscle development Nrk2b-deficient zebrafish [24]. DG is required for normal Paxillin concentration at the MTJ: DG-deficient zebrafish show reduced concentration of Paxillin at the MTJ. Addition of NAD+ improves Paxillin concentration at the MTJ and Paxillin overexpression reduces muscle degeneration in DG-deficient zebrafish [25]. In contrast, Paxillin overexpression does not rescue zebrafish deficient for Itga7 [25]. These data suggest that Paxillin functions downstream of $\mathrm{NAD}+$ to improve muscle resilience in DG-deficient zebrafish. However, whether Paxillin concentration at the MTJ is disrupted in zebrafish models of secondary dystroglycanopathy and whether Paxillin overexpression ameliorates muscle degeneration in zebrafish models of secondary dystroglycanopathy have not been investigated.

We used the $f k r p$ morphant model of FKRP-associated dystroglycanopathy [39, 72] to address unanswered questions regarding NAD+ regulation of the ECM in a secondary dystroglycanopathy. In addition, given the data indicating that there is not a strict correlation between DG glycosylation levels and phenotype, we investigated neuromuscular junction development in $f k r p$ morphants. Supplementing $f k r p$ morphants with $\mathrm{NAD}+$ at gastrulation improves 
laminin polymerization at the MTJ, muscle structure, and muscle function. Despite the fact that Paxillin localization is disrupted in $f k r p$ morphants, Paxillin overexpression failed to rescue any of these phenotypes. Early NMJ development was disrupted in $f k r p$ morphants and improved with NAD+. To our knowledge, this is the first report of initial NMJ formation being disrupted in an animal model of secondary dystroglycanopathies. As initial NMJ development has not been investigated in an animal model of DG deficiency, we analyzed early NMJ development in dag1 morphants. NMJ development is disrupted in dag1 morphants, although to a lesser extent than $f k r p$ morphants. In contrast to fkrp morphants, NAD+ supplementation did not improve NMJ development in dag1 morphants. Finally, we show that muscle-specific overexpression of $f k r p$ improved MTJ morphology but was not sufficient to improve muscle structure or function. Taken together, these data indicate that, at least in the zebrafish, FKRP-associated dystroglycanopathy does not phenocopy DG-deficiency. Furthermore, these data show that Fkrp is required for normal NMJ development and is required in tissues other than muscle.

\section{Methods}

\section{Zebrafish husbandry and transgenic lines}

All embryos were obtained from natural spawnings of adult zebrafish maintained on a 14-h light/10-h dark cycle at $28.5^{\circ} \mathrm{C}$. Embryos were reared in $1 \mathrm{X}$ embryo rearing medium (ERM) with methylene blue and staged according to [40]. The following strains were utilized/generated: $\mathrm{AB}, \mathrm{Tg}$ (fli1:EGFP) [42], $\mathrm{Tg}$ (ef1 $\alpha$ : $x b p 1 \delta$-GFP) [46] (a kind gift from Dr. Shao Jun Du), $T g$ (hsp70l:pxn-EGFP) [10], Tg (hsp70l:fkrp-EGFP), and $T g(-503 u n c: f k r p-E G F P)$. The Tg (hsp70l:fkrp-EGFP) and $T g(-503 u n c: f k r p-E G F P)$ lines were generated via the gateway cloning system. A sequence surrounding the fkrp gene was amplified with NCBI-verified primers and was gated and inserted into the pDONR221 vector (Invitrogen). The heat shock gateway $2225^{\prime}$ vector, GFP gateway $3663^{\prime}$ vector, and the gateway 394 destination vector [41] were used along with the donor vector and LR clonase II for the $\mathrm{Tg}$ (hsp70l:fkrp-EGFP) line. Components remained the same for the $T g(-503 u n c: f k r p-E G F P)$ line with the exception of the -503unc zebrafish muscle-specific promoter [5], which was used in place of the heat shock gateway $2225^{\prime}$ vector. Plasmids were injected at the single cell stage as previously described [24].

\section{MO injections}

Antisense MOs were obtained from Gene Tools, LLC, and hydrated at $65^{\circ} \mathrm{C}$ for $10 \mathrm{~min}$ with sterile water to generate 1-mM stocks. For fkrp morphant experiments, the previously published fkrp MO2 (5'-CTTGTGGTTTTA TGGCAGAAAGAGT-3') [39] was utilized and injected into the yolk of 1-2 cell stage embryos so that embryos received approximately $3.2 \mathrm{ng}$ of MO. For dag1 morphant experiments, the previously published dag1 MO (dag1 MO1) (5'-CATGCCTGCTTTTATTTTCCCTCGC-3' [53] and an additional slightly overlapping dag1 $\mathrm{MO}$ (dag1 MO2) (5'-CCCTCGCTCGTACAAAAGAGGA CGT-3') were co-injected into the yolk of 1-2 cell stage embryos so that embryos received approximately $12 \mathrm{ng}$ of each dag1 MO1 and dag1 MO2. Embryos utilized in experiments alongside NAD+- and EmergenC-treated embryos were separated into $60-\mathrm{mm}$ Petri dishes with 25 embryos per dish in $10 \mathrm{~mL} 1 \mathrm{X}$ ERM.

\section{NAD+ and EmergenC supplementation}

Fkrp morphants receiving EmergenC treatment (Alacer Corp) were separated into 60-mm Petri dishes with 25 embryos per dish in $10 \mathrm{~mL} 1 \mathrm{X}$ ERM-EmergenC solution. The NAD+ solution was prepared as previously described [24] except in sterile water. The EmergenC solution was diluted in 1X ERM so that the level of niacin in the solution was equal to $7.61 \mu \mathrm{M}$. Supplementation began at $6 \mathrm{~h}$ post fertilization (hpf) or $24 \mathrm{hpf}$ depending on the experiment, and the solution was changed every $24 \mathrm{~h}$ until embryo fixation.

\section{Expression of constructs with the heat shock promoter}

To constitutively overexpress Fkrp, uninjected and injected $T g$ (hsp70l:fkrp-EGFP) embryos were heat shocked at $38^{\circ} \mathrm{C}$ for $1.25-2 \mathrm{~h}$ at the 15 somite stage. To overexpress Paxillin, $\mathrm{Tg}$ (hsp70l:pxn-EGFP) were heat shocked at $38^{\circ} \mathrm{C}$ for $1.5 \mathrm{~h}$ at $12 \mathrm{hpf}$, then for $1.5-2 \mathrm{~h}$ daily until fixation. Heat-shocked fish were immediately transferred to $28^{\circ} \mathrm{C}$.

\section{Mobility assays}

Touch response analysis was performed at $3 \mathrm{dpf}$. Larvae were placed in 1X ERM and stimulated at the posterior end with fishing wire. The number of touches to evoke an escape response was recorded. For larvae that did not respond after 50 touches, 50 was recorded as their touches to response.

\section{Phalloidin staining, bungarotoxin staining, and immunohistochemistry}

All embryos were fixed in 4\% Paraformaldehyde (PFA) for $2-4 \mathrm{~h}$ at room temperature, depending on the stage. After fixation, embryos received five rinses in PBS-0.1\% Tween 20. Unless co-stained with alpha-bungarotoxin, embryos stained with phalloidin were permeabilized for $1.5 \mathrm{~h}$ in PBS-2\% Triton-X-100, followed by incubation in $1 / 20$ phalloidin-546 (Invitrogen) in PBS-2\% Triton-X-100 or PBS$0.1 \%$ Tween 20 overnight at $4{ }^{\circ} \mathrm{C}$. Embryos stained with 
alpha-bungarotoxin (Molecular Probes) and SV2 were permeabilized in $1 \mathrm{mg} / \mathrm{ml}$ collagenase in $1 \mathrm{X}$ PBS for 75 min, then were stained with 1/500 alpha-bungarotoxin-647 for $2-4 \mathrm{~h}$ at room temperature. For co-staining, 1/20 phalloidin-546 was added to the alpha-bungarotoxin solution in antibody block. All embryos were then subjected to five rinses for $5 \mathrm{~min}$ each in PBS-0.1\% Tween 20, followed by an overnight incubation in antibody block (5\% BSA, 1\% DMSO, 1\% Triton-X-100, 0.2\% saponin in 1X PBS) for embryos receiving subsequent immunohistochemistry treatments. Primary antibodies were added at a concentration of 1/50 (anti-laminin (Thermo Scientific), anti-SV2 (Developmental Studies Hybridoma Bank), anti-Paxillin (BD Biosciences), anti-beta-DG (Novocastra-Leica)) in antibody block and incubated for 2-8 h at room temperature followed by overnight at $4{ }^{\circ} \mathrm{C}$. Embryos treated with anti-SV2 were incubated for 3 days at $4{ }^{\circ} \mathrm{C}$ following the initial overnight incubation. All embryos were then treated with antibody block for $8 \mathrm{~h}$ at room temperature or overnight at $4{ }^{\circ} \mathrm{C}$, followed by an overnight incubation $4{ }^{\circ} \mathrm{C}$ plus $2-6 \mathrm{~h}$ at room temperature in secondary antibody (Invitrogen) diluted 1/150-1/200 in block. Embryos were rinsed out of secondary antibody with PBS-0.1\% Tween 20 prior to deyolking, mounting, and imaging.

\section{Imaging}

Fixed and stained embryos were deyolked, then mounted and imaged in $80 \%$ glycerol. Fluorescent images of fixed, stained fish were captured at $\times 20$ with an Olympus Fluoview IX-81 inverted microscope with FV1000 confocal system or at $\times 25$ with a Leica DMi8 confocal microscope. Images for $\mathrm{Tg}(e f 1 \alpha: x b p 1 \delta$-GFP) were acquired at $\times 10$ on a Zeiss Axio Imager Z1 microscope with a Zeiss ApoTome or at $\times 5$ on a Zeiss microscope with Vivatome attachment. Exposure time was kept consistent between all embryo groups within an experiment.

\section{Statistical analysis}

All data were log transformed in GraphPad PRISM 8 before performing statistical comparisons. All subsequent analyses were performed in GraphPad PRISM 8. Statistical comparisons of data were performed using a twotailed student $t$ test for comparisons of two groups, and an ordinary one-way ANOVA with Tukey's ad hoc analysis for assays with three or more groups. Categorical data were scored in using Fisher's exact test for two categories and the chi square test for three or more categories.

\section{Image analysis}

Muscle fiber degeneration was quantified by counting the number of muscle segments with degeneration per embryo and calculating the percent of myotomes with muscle degeneration. MTJ angles were analyzed in FIJI by using the angle tool to measure the angles formed by the MTJ. For laminin and beta-DG staining analyses, images were blinded using a Perl script and embryos were scored according to their relative staining intensity (Additional file 1: Figure S1). Intersegmental vessel (ISV) lengths were measured in FIJI using the segmented line tool.

For anisotropy analysis, a polygon was drawn in eight myotomes per image analyzed using the polygonal lasso tool in Adobe Photoshop. The $x, y$ coordinates of each polygon were recorded. Masks were generated from the coordinates, and 2DWTMM analysis was performed on each of these masks as previously described [24, 25].

For analysis of $x b p 1$ transgenic embryos, fluorescence intensity was quantified using FIJI. A polygon was drawn around the dorsal muscle above the notochord for all embryos in the trunk region. The average pixel intensity (mean gray value) was calculated. The mean average pixel intensity for all controls was calculated. These values were used to determine the percent average pixel intensity for all embryos. Images for all $x b p 1$ transgenic embryos were blinded using a Perl script prior to analysis.

For NMJ analyses, confocal images were processed into maximum intensity projections of fast-twitch (distributed) and slow-twitch (myoseptal) innervation using FIJI and were subsequently blinded using a Perl script. Masks for the fish, horizontal myoseptum, and myoseptal innervation were generated using FIJI. The masks were imported into MATLAB (Mathworks) and used to segment acetylcholine receptor (AChR) and Synaptic vesicle protein 2 (SV2) fluorescence channels. The fluorescence images were enhanced using adaptive histogram equalization (MATLAB "adapthisteq" function) and then denoised with a 1-pixel radius gaussian filter (MATLAB “imguassfilt" function). Single masks of the fluorescence channels were then generated using a threshold of 30 on each channel, which were then combined with an "or" statement. Thresholds up to 58 and down to 10 yielded the same trend in the data, indicating that the measurements chosen were not overly sensitive to this parameter. The masks were skeletonized, and subsequently cleaned and despurred using the MATLAB "bwmorph" command. Branchpoints were also identified using the MATLAB "bwmorph" command. The fish, horizontal myoseptum, and myoseptal innervation masks were used to define the muscle segments, and the indicated measurements were performed for each muscle segment across all embryos analyzed.

\section{Results}

NAD+ supplementation prior to muscle development improves muscle and MTJ structure

Injection of previously published fkrp MOs recapitulated the previously described phenotype of $f k r p$ morphants 


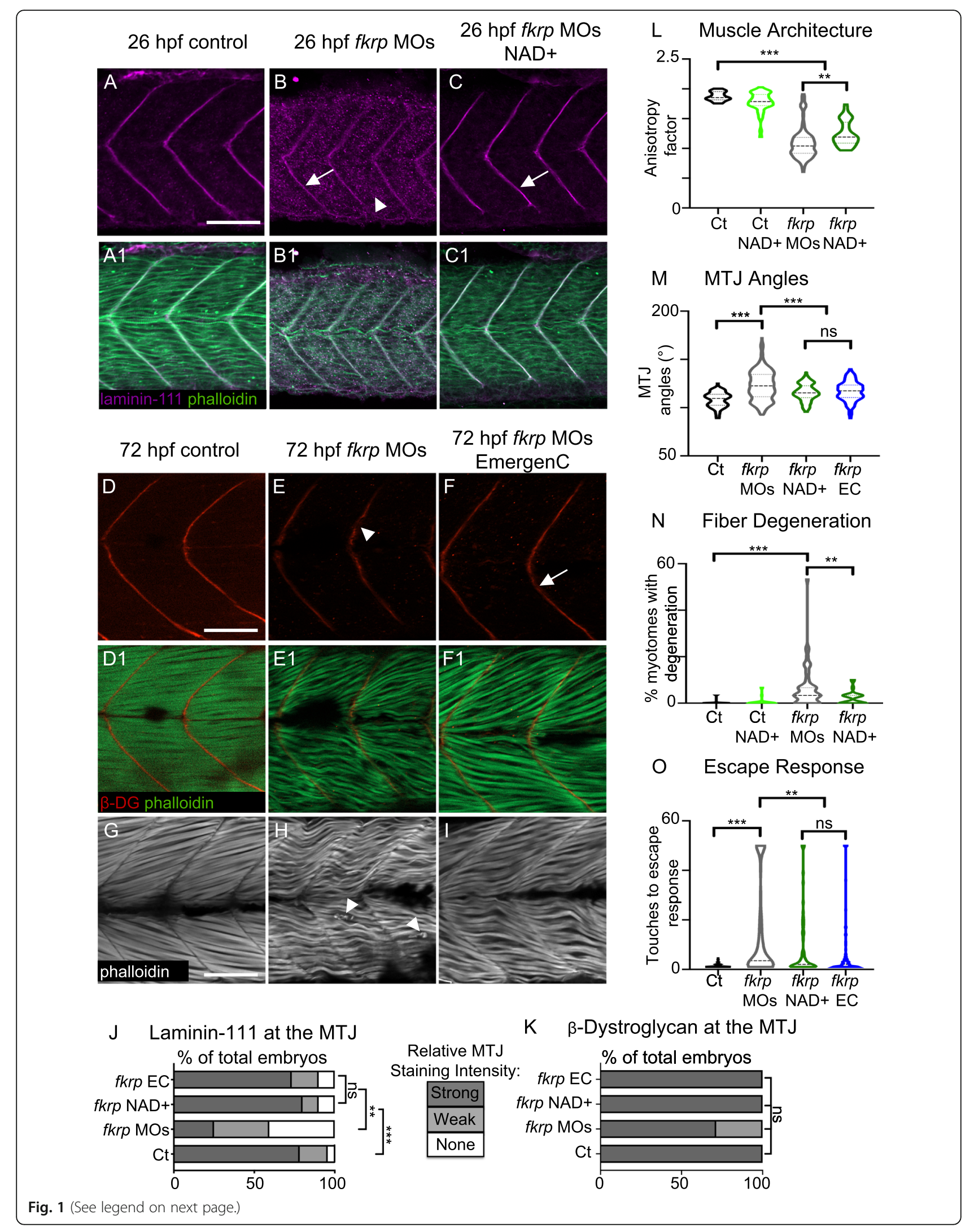


(See figure on previous page.)

Fig. $1 \mathrm{NAD}+$ or EmergenC supplementation at gastrulation improves muscle structure and function in fkrp morphants. (A-C) Anterior left, dorsal top, side-mounted embryos at 26 hpf stained for laminin-111 (purple) and actin (phalloidin, green). (A, A1) Control embryo. Laminin is concentrated at the MTJ. (B, B1) fkrp morphant. Although laminin is present at the MTJ (white arrow), it is also present within the myotome (white arrowhead). (C, C1) fkrp morphant treated with $0.1 \mathrm{mM} \mathrm{NAD+}$ at 6 hpf. Laminin is concentrated at the MTJ (white arrow) as in control embryos. (D-F1) Anterior left, dorsal top, side-mounted embryos at $72 \mathrm{hpf}$ stained for beta-DG (red) and f-actin (phalloidin, green). (D, D1) Control embryo. (E, E1) fkrp morphant. Beta-DG staining appears weaker at the MTJ (white arrowhead). (F, F1) fkrp morphant treated with EmergenC at 6 hpf. Beta-DG staining appears stronger at the MTJ (white arrow). (G-I) Anterior left, dorsal top, side-mounted embryos at 72 hpf stained for f-actin (phalloidin, gray). (G) Control embryo. (H) fkrp morphant. White arrowheads indicate single detached fibers. (I) fkrp morphant treated with EmergenC at 6 hpf. Note the improved muscle fiber structure. (J) Relative staining intensity of laminin-111 at the MTJ in 26 hpf embryos, based on no staining/localization (none, white), weak staining/localization (weak, light gray), and strong staining/localization (strong, dark gray) (images were blinded prior to analysis, see the "Methods" section). Controls ( $n=23$ embryos) have greater laminin intensity staining than fkrp morphants ( $n=32$ embryos). NAD+ $(n=10$ embryos) and EmergenC ( $n=30$ embryos) supplementation improve laminin-111 concentration at the MTJ in fkrp morphants. (K) Relative staining intensity of beta-DG at the MTJ in 72 hpf embryos, based on weak staining (weak, light gray) and strong staining (strong, dark gray). Although fkrp morphants have more embryos with weaker beta-DG staining at the MTJ ( $n=7$ embryos), there is no significant difference in beta-DG at the MTJ between untreated morphants, morphants treated with NAD $(n=7$ embryos), EmergenC ( $n=7$ embryos), or control morphants ( $n=5$ embryos). (L) Quantification of muscle organization at $72 \mathrm{hpf}$. The anisotropy factor in embryos injected with fkrp MOs ( $n=96$ half-myotomes) is reduced compared to uninjected controls ( $n=16$ half-myotomes), and NAD+ ( $n=40$ half-myotomes) significantly increases the anisotropy factor in fkrp morphants. (M) Quantification of MTJ angles at 72 hpf. Injection of fkrp MOs ( $n=343$ MTJs) significantly increases MTJ angles compared to uninjected controls ( $n=98$ MTJs). Either NAD+ $(n=105$ MTJs) or EmergenC ( $n=158$ MTJs) treatment significantly reduces MTJ angles compared to untreated morphants. (N) Untreated fkrp morphants $(n=54$ embryos) have a significantly higher percent of myotomes per embryo with fiber detachments than controls $(n=40$ embryos) at $72 \mathrm{hpf}$. NAD+ ( $n=31$ embryos) supplementation significantly reduces the percent of myotomes with dystrophy per embryo. (0) Injection of fkrp MOs ( $n=184$ embryos) significantly increases the number of touches required to induce an escape response compared to uninjected controls ( $n=185$ embryos). NAD+ ( $n=84$ embryos) or EmergenC ( $n=111$ embryos) treatment significantly reduces the number of touches to invoke an escape response. Scalebars are $50 \mu \mathrm{m} .{ }^{*} p<0.05,{ }^{* *} p<0.01,{ }^{* * *} p<0.001$, ns non-significant

$[39,72]$ and the recently described fkrp mutant [63]. In agreement with previous findings, multiple aspects of muscle development are disrupted in $f k r p$ morphants: MTJs are significantly wider than in control embryos (Fig. 1M), muscle fibers are disorganized (Fig. 1L), and muscle degeneration is observed (Fig. $1 \mathrm{H}, \mathrm{N}$ ). Muscle fiber adhesion to the MTJ and ECM organization at the MTJ are disrupted in $f k r p$ morphants $[39,72]$. We also found that laminin (Fig. 1A, B, J) is reduced in $f k r p$ morphants compared to controls and that beta-DG is concentrated at the MTJ, although sometimes slightly reduced (Fig. 1D, E, K). We next asked whether muscle function was disrupted by quantifying the number of touches required to induce an escape response. We found that fkrp morphants have reduced muscle function in that they require significantly more touches to induce an escape response (Fig. 1O). These data indicate that $f k r p$ is necessary for normal muscle development and function.

We previously showed that either NAD+ or EmergenC supplementation at gastrulation is sufficient to improve ECM organization, reduce muscle degeneration, and improve motility in dag1 morphants [25]. We hypothesized that $\mathrm{NAD}+/$ EmergenC increased laminin organization by potentiating clustering of the other major laminin receptors in muscles, Integrins alpha6/beta1 and alpha7/beta1. We asked if $\mathrm{NAD}+/$ EmergenC is sufficient to improve laminin organization in the context of impaired DG glycosylation because hypoglycosylated DG could hinder increased laminin organization. $\mathrm{NAD}+$ or EmergenC supplementation at gastrulation increased the concentration of laminin at the MTJ (Fig. 1C, J), decreased MTJ angles (Fig. 1M), decreased muscle degeneration (Fig. 1N), improved muscle fiber organization (Fig. 1L), and significantly reduced the touches required to evoke an escape response (Fig. 1O). These data indicate that $\mathrm{NAD}+$ or EmergenC supplementation during gastrulation is sufficient to improve muscle development and function in Fkrp-deficient zebrafish.

\section{NAD+/EmergenC are not sufficient to improve abnormal vascularization, midbrain-hindbrain development, or the increased unfolded protein response in fkrp morphants} Fkrp is required for normal zebrafish vascularization: $f k r p$ morphants and the recently described fkrp mutant exhibit shorter intersegmental vessel (ISV) lengths compared to wild-type embryos [63, 78]. Fli1:EGFP transgenics injected with fkrp MOs exhibited shorter ISV lengths (including truncated vessels) than uninjected controls (Fig. 2E, F, H). NAD+ supplementation had no significant effect on ISV length in $f k r p$ morphants (Fig. 2G, H). We also analyzed ISV length when normalized to the width of the embryos because muscle development is disrupted in fkrp morphants. The same result was observed with normalized data (not shown). These data suggest that: (1) NAD+ supplementation is not sufficient to improve vascularization in $f k r p$ morphants, and (2) the vascularization defects observed in $f k r p$ morphants are likely a consequence of $f k r p$ knockdown 


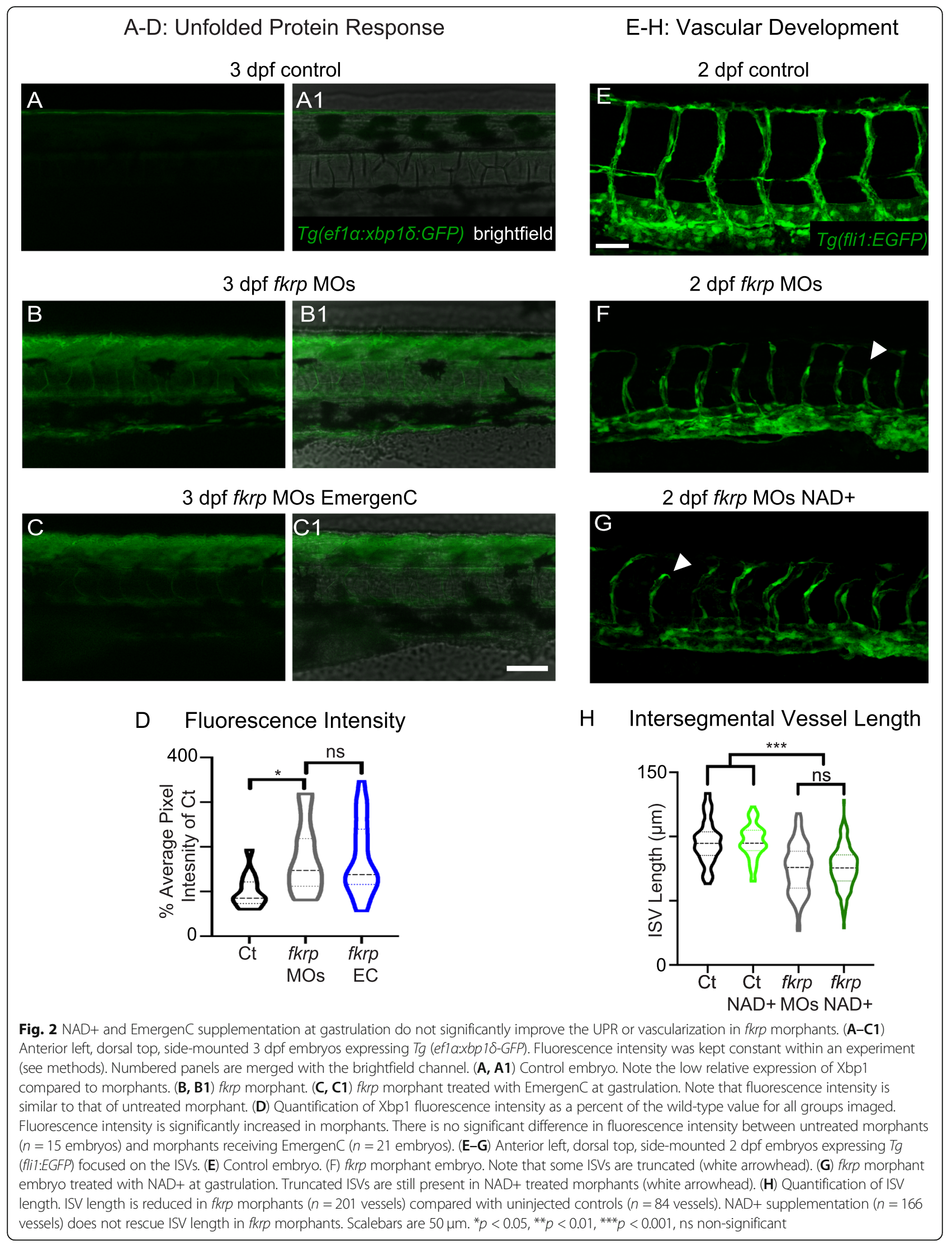




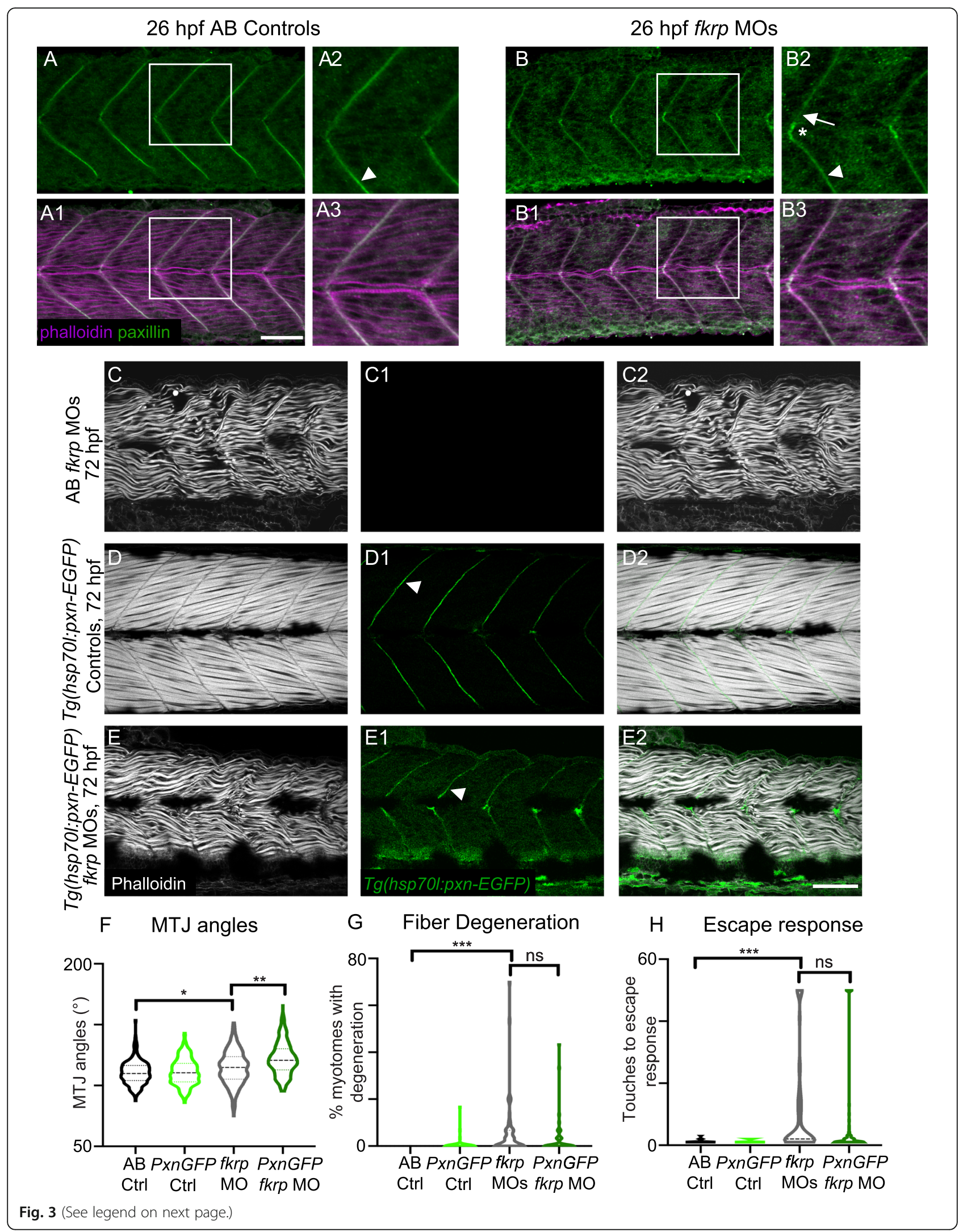




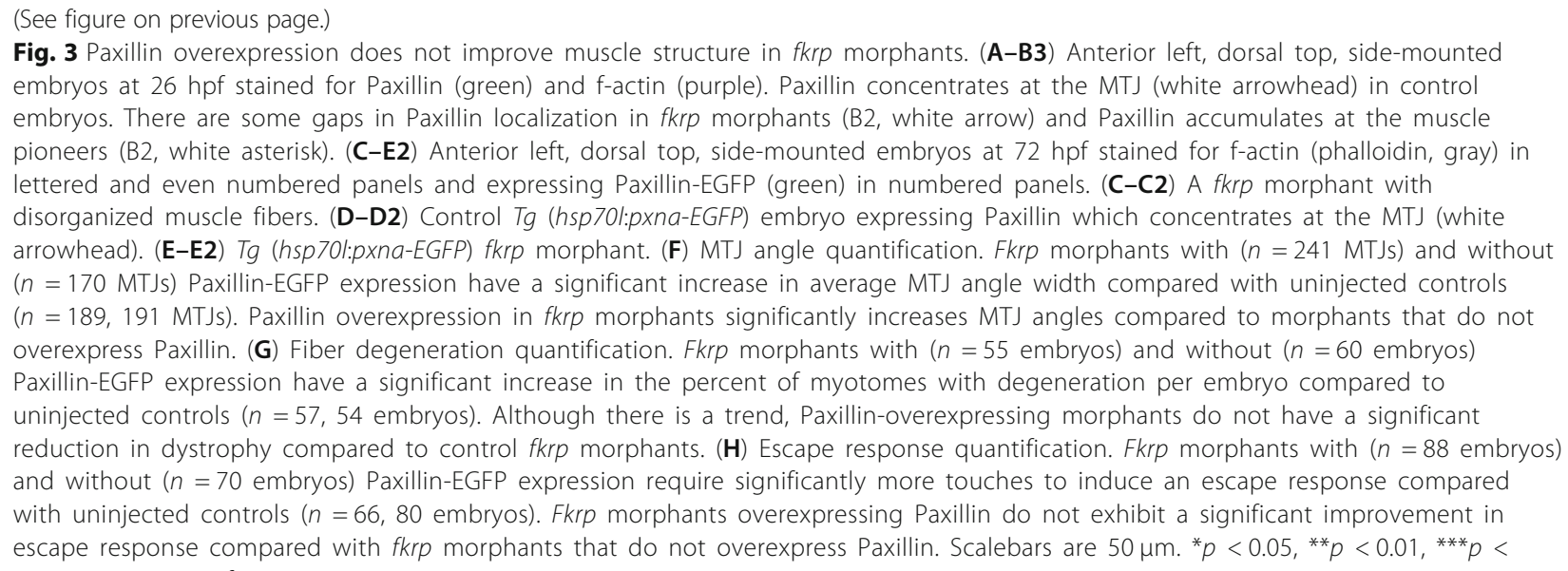

and not aberrant muscle development and/or homeostasis.

Zebrafish deficient for Fkrp exhibit abnormal midbrain-hindbrain boundary formation that has been likened to cobblestone lissencephaly [72]. We also observed abnormal midbrain-hindbrain boundary formation in $f k r p$ morphants at $26 \mathrm{hpf}(n=8)$ compared with uninjected controls. NAD+ was not sufficient to rescue midbrain-hindbrain boundary formation $(n=8$, data not shown). Zebrafish models of FKRP-associated dystroglycanopathy are also associated with endoplasmic reticulum (ER) stress and activation of the unfolded protein response (UPR) [47]. One marker of the UPR is increased activation of the $x b p 1$ transcript, which is upregulated in $f k r p$ morphants [47] and the recently described fkrp mutant [63]. To determine whether EmergenC could improve ER stress in $f k r p$ morphants, we injected $f k r p$ MOs into the $T g$ (efl $\alpha$ : $x b p 1 \delta$-GFP) line that allows visualization of $x b p 1$ activation [46]. Injection of $f k r p$ MOs increased the UPR (Fig. 2B) compared with controls (Fig. 2A). However, untreated and EmergenC-treated fkrp morphants did not significantly differ in relative fluorescence intensity (Fig. 2C, D). Taken together, these results suggest that the benefits of NAD+ supplementation may be limited to muscle in Fkrp-deficient zebrafish.

\section{Paxillin overexpression does not significantly reduce muscle degeneration}

Paxillin is an Integrin-associated protein that is required for cell adhesion to the ECM [74]. Paxillin concentrates at MTJs in wild-type muscle ([14], Fig. 3). Paxillin plays a role in the Nrk2b-NAD+-laminin cell adhesion pathway. Paxillin concentration at the MTJ is disrupted in $n r k 2 b$ morphants and rescued with NAD+ [24]. Paxillin overexpression rescues $n r k 2 b$ morphants [24]. Similarly, concentration of Paxillin at the MTJ is disrupted in dag1 morphants and is improved with NAD+. Paxillin overexpression improves muscle structure in dag1 morphants [25]. It is not known if either Paxillin concentration at the MTJ is disrupted in animal models of secondary dystroglycanopathy or if Paxillin overexpression improves muscle structure in these models. Paxillin concentration at the MTJ was disrupted in 26 hpf fkrp morphants (Fig. 3B) compared with controls (Fig. 3A). Paxillin does concentrate at the center of the MTJ adjacent to muscle pioneers that are slow-twitch fibers (Fig. 3B at the crux of the vshaped MTJ, this was observed in 14/16 fkrp morphants). However, Paxillin concentration at the MTJ adjacent to fast-twitch muscle is disrupted (Fig. 3B2, arrow). These data suggest that Fkrp is required for normal development of the MTJ adjacent to fast-twitch muscle. Overexpression of Paxillin was sufficient for Paxillin to concentrate at the fast-muscle MTJ in fkrp morphants (Fig. 3E1 arrowhead). However, in contrast to what was previously observed in dag1 morphants [25], Paxillin overexpression did not reduce muscle degeneration or improve the escape response in $f k r p$ morphants (Fig. 3G-H). Paxillin overexpression actually increased MTJ angles (Fig. 3F). These data indicate that, in contrast to dag1 morphants, Paxillin overexpression is not sufficient to improve muscle structure in $f k r p$ morphants.

\section{NAD+ supplementation after initial muscle development improves MTJs but not muscle structure}

$\mathrm{NAD+/EmergenC}$ supplementation prior to muscle development is sufficient to improve muscle structure and function in dag1 [25] and fkrp morphant embryos (Fig. 1). It is not known whether NAD+ or EmergenC can improve muscle structure and function after initial muscle development. We supplemented fkrp morphants with NAD+ or EmergenC at $24 \mathrm{hpf}$ (after initial muscle development). Both NAD+ and EmergenC supplementation significantly improved MTJ angles (Fig. 4f). In contrast, neither NAD+ 


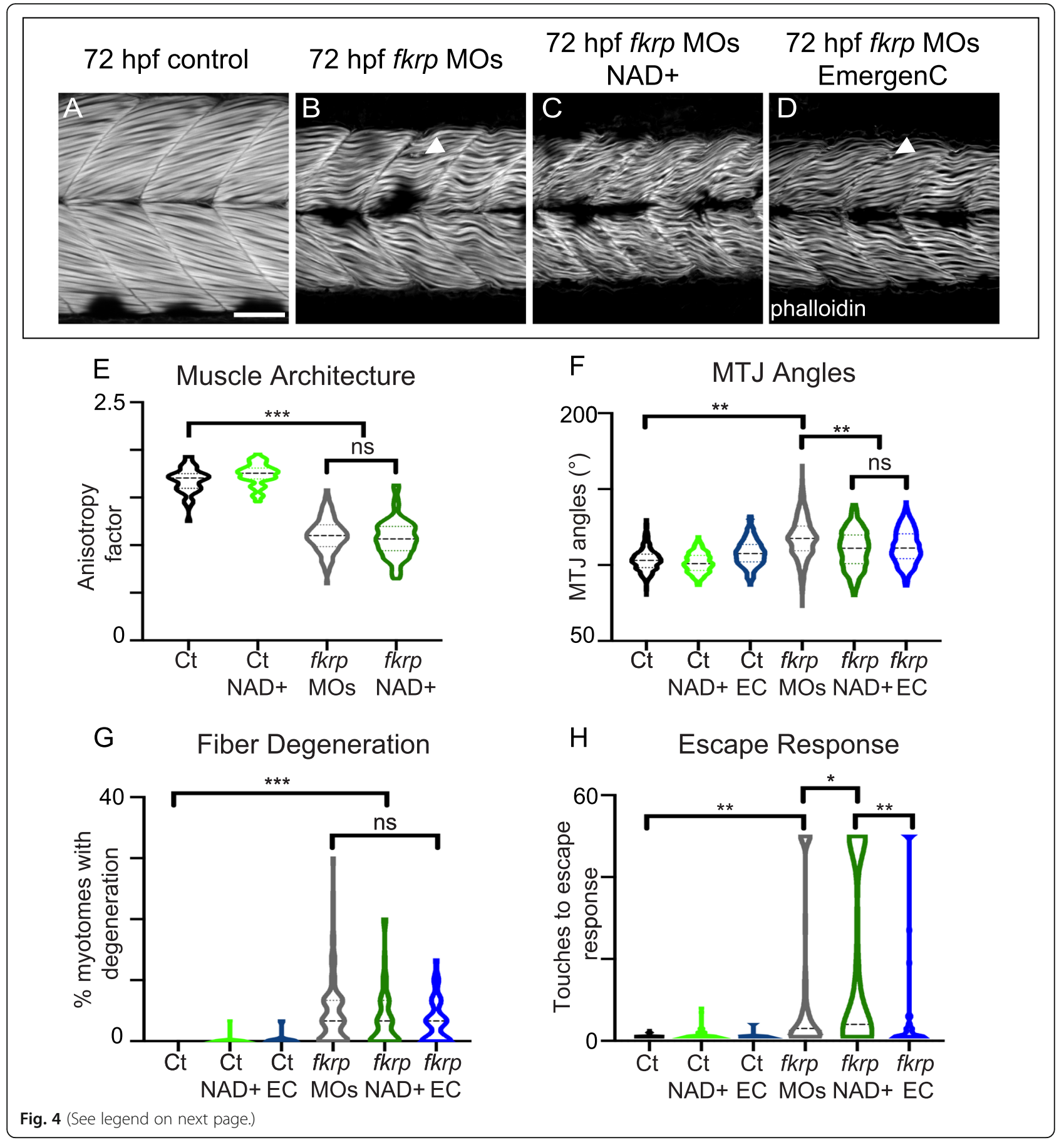




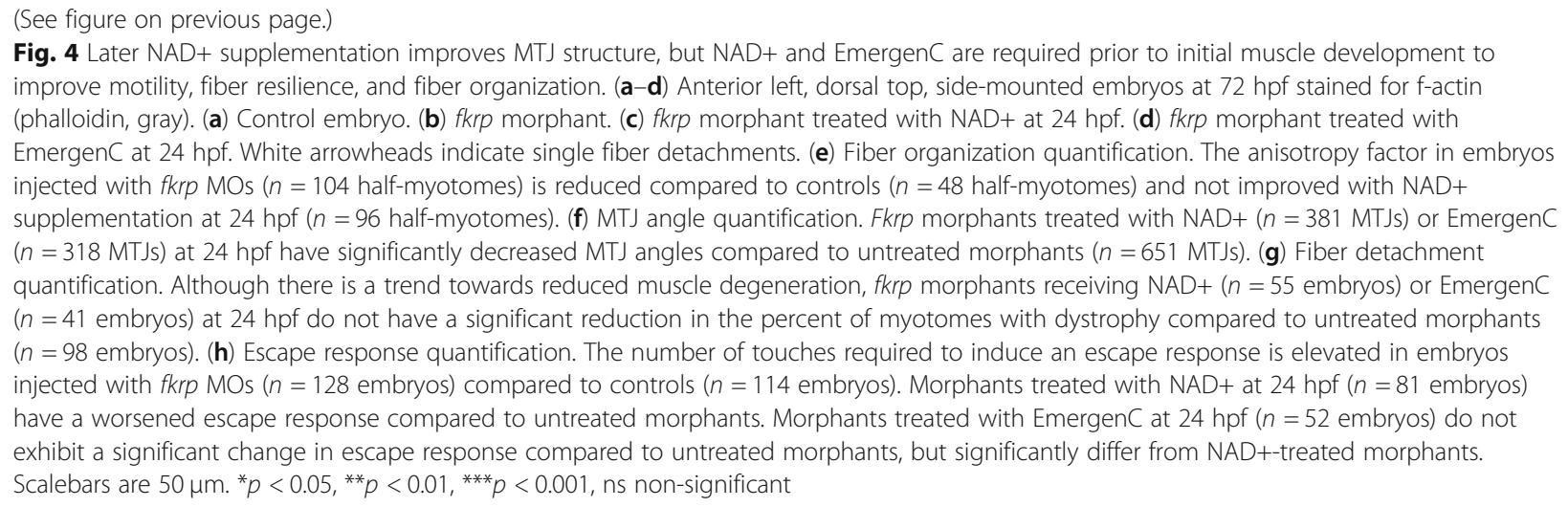

nor EmergenC supplementation at $24 \mathrm{hpf}$ was sufficient to improve muscle fiber organization, reduce muscle degeneration (Fig. $4 \mathrm{a}-\mathrm{d}, \mathrm{e}, \mathrm{g}$ ), or improve the escape response (Fig. 4h). Thus, NAD+/EmergenC supplementation after initial muscle development is not sufficient to ameliorate muscle degeneration and improve muscle function in Fkrp-deficient zebrafish embryos.

\section{NMJ development is disrupted and partially improved with NAD+ supplementation in fkrp morphants}

The DGC is necessary for NMJ maturation after birth in mouse [32, 35, 43]. DG glycosylation is also necessary for normal NMJ formation during muscle regeneration [22]. It is generally thought that DG is not required for NMJ development but is required for NMJ maturation/stabilization/regeneration [27]. We asked whether initial NMJ development is disrupted in secondary dystroglycanopathies. NMJ structure at $72 \mathrm{hpf}$ was analyzed by staining for SV2 and postsynaptic AChRs (alpha-bungarotoxin). We focused on analysis of innervation of fast-twitch fibers (distributed innervation, see skeletons Fig. 5). There is an extensive network of distributed NMJs in 72 hpf control embryos (Fig. 5A). This network is qualitatively disrupted in fkrp morphants where there appeared to be shorter chains of NMJs (Fig. 5B). Supplementation of NAD+ or EmergenC improved NMJ length, although NMJs were not fully restored (Fig. 5C, D). In order to quantify innervation, we developed a semi-automated technique in MATLAB to skeletonize NMJs (see methods). This analysis showed that although branching frequency was normal, skeleton length was reduced in $f k r p$ morphants (Fig. 5E, F). NAD+ or EmergenC supplementation at 6 hpf slightly, but significantly, improved skeleton length in fkrp morphants (Fig. 5E). These results suggest that glycosylated DG is necessary for innervation, but not branching.
As mentioned above, DG contributes to NMJ maturation and stabilization [27]. Given the above data showing that initial NMJ development is disrupted in $f k r p$ morphants, we asked whether DG is also required for NMJ development. Skeleton length was slightly, but significantly, reduced in dag1 morphants (Fig. 6B) compared to controls (Fig. 6A). However, NAD+ supplementation at gastrulation did not significantly increase skeleton length in dag1 morphants despite rescuing other aspects of the phenotype (Fig. 6C, D, F). This result contrasts with what we observed in $f k r p$ morphants supplemented with NAD+. Thus, we compared the relative severity of NMJ skeleton length defects in fkrp morphants versus dag1 morphants. On average, dag1 morphant skeleton length was $83.9 \%$ of that of uninjected controls. In contrast, fkrp morphant skeleton length on average was $51.4 \%$ of that of uninjected controls (Fig. 6G). Thus, NMJs are more severely disrupted in fkrp morphants compared to dag1 morphants.

Somewhat surprisingly, we found that NAD+ supplementation at $24 \mathrm{hpf}$ actually significantly worsened the escape response in $f k r p$ morphants (Fig. $4, p<0.05$ ) despite the fact that we did not observe a significant deterioration of muscle structure. Thus, we asked how NAD+ supplementation at $24 \mathrm{hpf}$ affected NMJ structure (Fig. 7). The effects of NAD+ on NMJ structure correlated with the motility defects: NAD+ at $24 \mathrm{hpf}$ resulted in significantly shorter skeleton lengths (Fig. 7E). There was no significant difference between $24 \mathrm{hpf}$-treated and untreated morphants in terms of branching frequency (Fig. 7F).

\section{Muscle-specific expression of fkrp is not sufficient to rescue fkrp morphants}

The above data indicate that Fkrp is necessary for early NMJ development and that disruption in NMJ morphology with $24 \mathrm{hpf} \mathrm{NAD}+$ treatment correlates with worse motility. These results raise the possibility that Fkrp function is required in non-muscle tissues. We tested this hypothesis by expressing Fkrp in a musclespecific manner. To confirm that constitutive Fkrp 


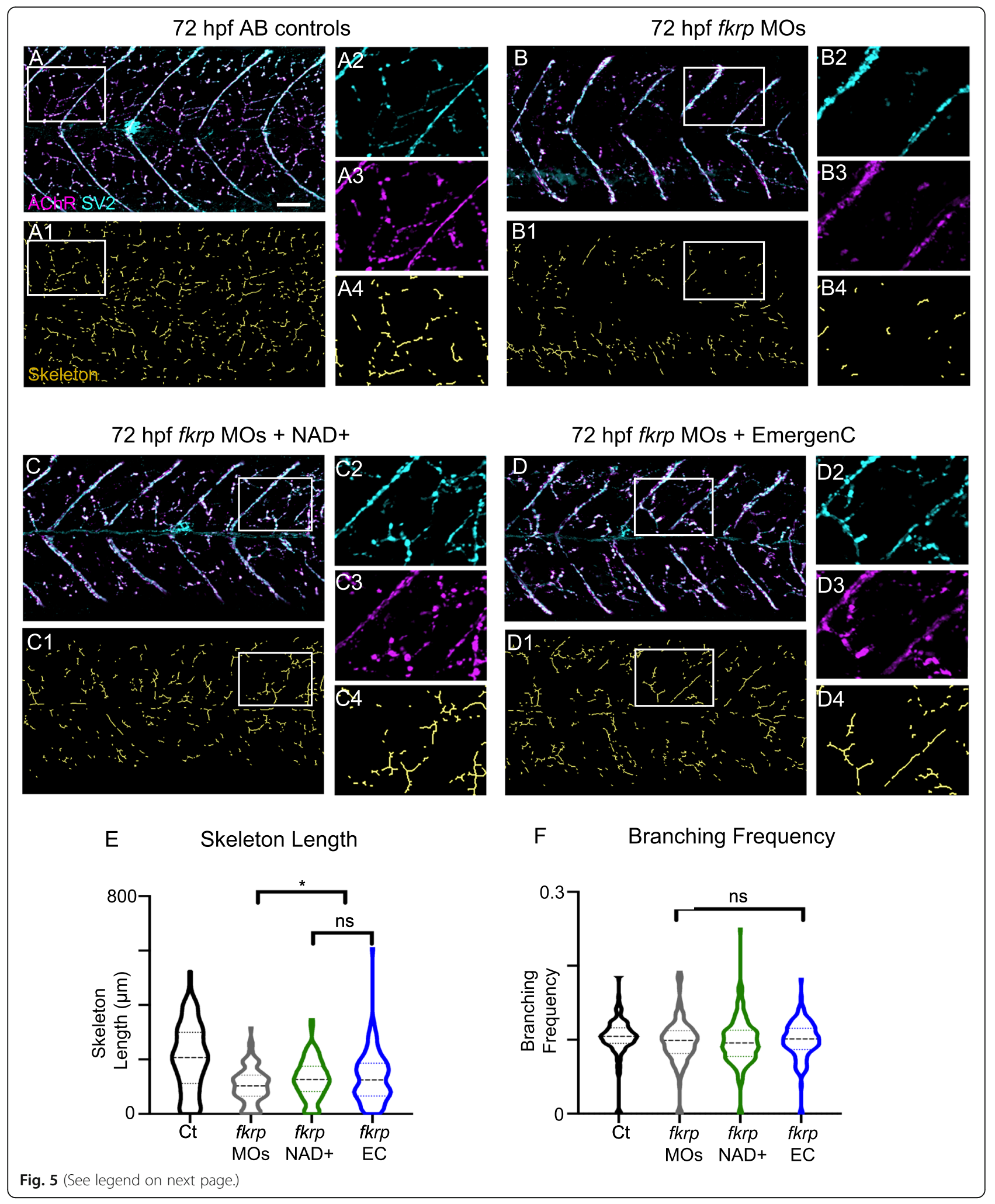


(See figure on previous page.)

Fig. 5 Fkrp morphants exhibit NMJ defects and NAD+ and EmergenC treatment prior to muscle development improves NMJ development. (AD4) Anterior left, dorsal top, side-mounted embryos at $72 \mathrm{hpf}$ with labeled AChR and SV2. (Lettered panels) Merged channels of AChR and SV2. (1) Skeletonized images. (2) Magnification of SV2 channel. (3) Magnification of AChR channel. (4) Magnification of skeleton channel. (A-A4) Control embryo. (B-B4) fkrp morphant embryo exhibiting reduced distributed innervation within the myotome. (C-C4) fkrp morphant embryo treated with NAD+ at 6 hpf shows increased NMJs. (D-D4) fkrp morphant embryo treated with EmergenC at 6 hpf also shows increased NMJs. (E) Length of skeletons. Skeleton length is reduced in fkrp morphants ( $n=153$ half-myotomes) compared to controls ( $n=144$ half-myotomes) but significantly increased in fkrp morphants receiving NAD+ ( $n=183$ half-myotomes) or EmergenC ( $n=127$ half-myotomes) at 6 hpf. (F) Degree of branching within the myotome in control embryos ( $n=141$ half-myotomes), fkrp morphants ( $n=147$ half-myotomes), and fkrp morphants receiving NAD+ ( $n=179$ half-myotomes) or EmergenC ( $n=126$ half-myotomes) at 6 hpf. Scalebars are $50 \mu m .{ }^{*} p<0.05,{ }^{* *} p<0.01,{ }^{* * *} p<0.001$, ns non-significant

overexpression could rescue fkrp morphants, we injected fkrp MOs into a Tg (hsp70l:fkrp-EGFP) line we generated (Fig. 8A). Previous data suggest that overexpression of $f k r p$ can be deleterious [77]. Although we observed a slight increase in MTJ angles (Fig. 8C), we did not observe any significantly adverse effects of Fkrp-EGFP on fiber degeneration or the escape response in control embryos (expression was induced at the 15 somite stage) (Fig. 8A, D, E). Global overexpression of Fkrp-EGFP in fkrp morphants improved fiber resiliency, MTJ angles, and the escape response compared to control morphants (Fig. 8B-E). These data indicate that (1) overexpression of Fkrp is not toxic under these conditions and (2) EGFP does not deleteriously affect Fkrp function. We next asked whether muscle-specific expression was sufficient to rescue the phenotype. We generated a transgenic line expressing Fkrp-EGFP under control of the -503unc promoter [5]. Muscle-specific overexpression of Fkrp did not affect muscle morphology in control embryos (Fig. 8F-F2). Muscle-specific overexpression of Fkrp in fkrp morphants decreased MTJ angles compared to EGFP negative control morphants (Fig. 8I). However, no other metrics of muscle structure/function were improved. Muscle-specific overexpression neither ameliorated fiber detachment (Fig. 8G, H, J) nor reduced touches required to induce an escape response (Fig. 8K). These data indicate that expression of Fkrp in muscle is not sufficient to rescue muscle morphology or function.

\section{Discussion}

Dystroglycanopathies are a relatively understudied subset of muscular dystrophies. One aspect of dystroglycanopathies that is not well understood is how the myomatrix is impacted. Whether strategies that improve the myomatrix and muscle structure in a primary dystroglycanopathy (DG deficiency) are efficacious in secondary dystroglycanopathies are also not known. We previously showed that either NAD+ supplementation or overexpression of an Integrin-associated adaptor protein (Paxillin) are sufficient to improve muscle structure and function in DG-deficient zebrafish. Here, we show that muscle phenotypes in a zebrafish model of FKRP-associated dystroglycanopathy are improved with NAD+ supplementation but not Paxillin overexpression. Although the mechanisms are not known, these data clearly indicate that primary and secondary dystroglycanopathies show some differences. Our data also suggest that there are differences even within secondary dystroglycanopathies. Whereas muscle-specific expression of Large is sufficient to rescue muscle structure and function in Large/myd mutant mice [27], we found that muscle-specific expression of $f k r p$ is not sufficient to improve muscle structure/function in fkrp morphant zebrafish. Finally, our data show that NMJ formation is disrupted earlier in development than has previously been observed in a primary and secondary dystroglycanopathy model. Taken together, these data indicate that it is necessary to study the secondary dystroglycanopathies individually within the context of the group as a whole.

\section{Cell-matrix adhesion and secondary dystroglycanopathy}

Adhesion of muscle fibers to the MTJ is necessary for muscle development and homeostasis. We previously identified a cell adhesion pathway that contributes to laminin organization at the MTJ: Nrk2b-mediated NAD+ production potentiates laminin organization at the MTJ during zebrafish muscle development [24]. We then demonstrated that exogenous NAD+ is sufficient to improve laminin organization, muscle structure, and muscle function in zebrafish deficient for either DG or Itga7 [25]. DG and Itga7 are both transmembrane receptors for laminin. We hypothesized that NAD+ improved laminin organization, at least in part, by increasing clustering of the remaining receptor (DG in Itga7-deficient embryos, and Itga7 in DG-deficient embryos). Here, we asked whether laminin organization would be increased if DG is present but hypoglycosylated. The rationale was that it is possible that the presence of hypoglycosylated DG that cannot bind laminin could inhibit the improvement of laminin organization with NAD+. The ribitol 5-phosphate transferase FKRP is necessary for glycosylation of DG $[21,38]$. We asked if NAD+ was sufficient to improve cell-matrix adhesion in fkrp morphant zebrafish embryos. NAD+ supplementation prior to muscle development was sufficient to improve laminin polymerization, muscle structure, and the escape response. 


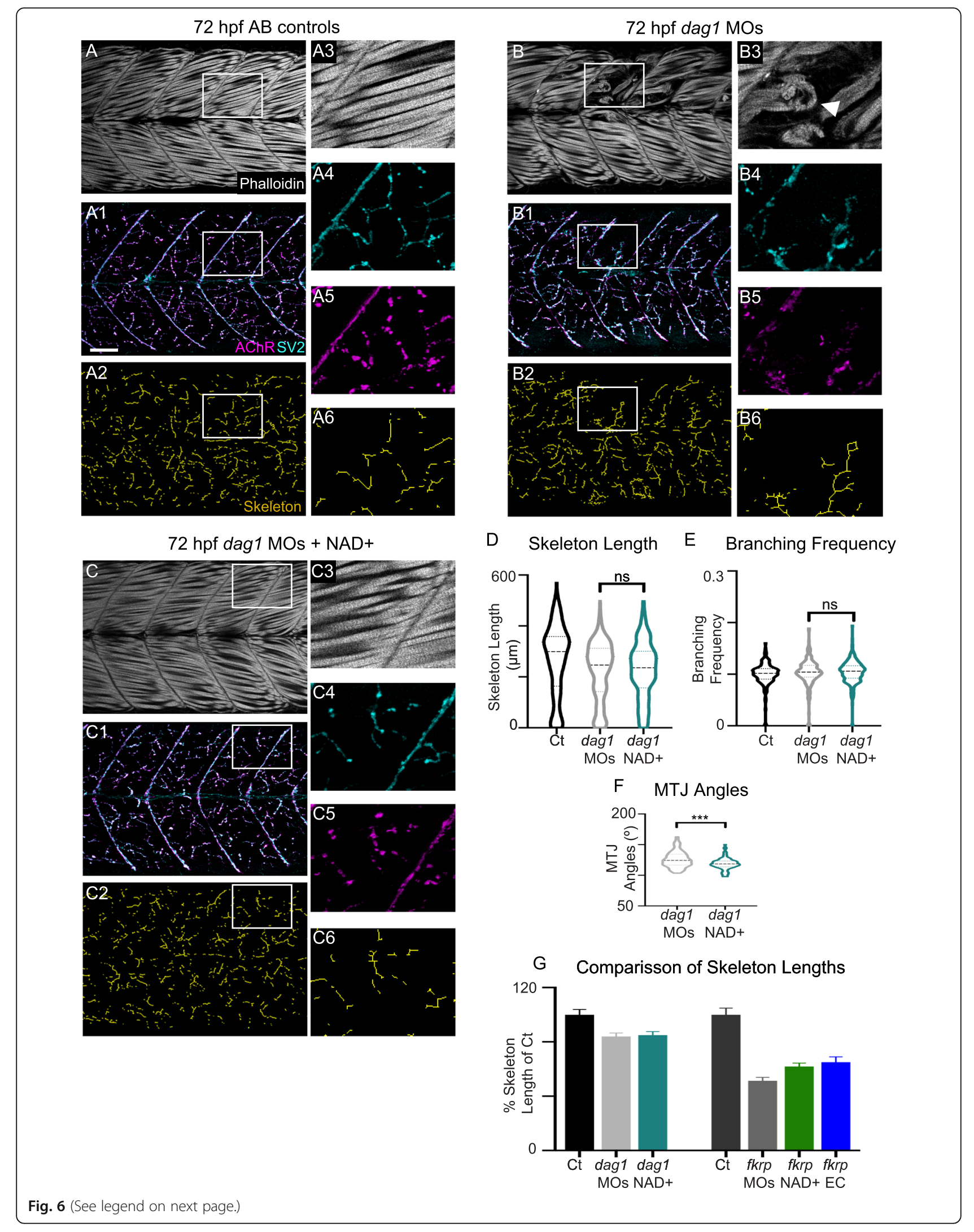


(See figure on previous page.)

Fig. 6 Skeleton length is less disrupted in dagl morphants than in fkrp morphants and is not significantly improved with NAD+ supplementation. (A-C6) Anterior left, dorsal top, side-mounted embryos at $72 \mathrm{hpf}$ with labeled actin, AChRs, and SV2. (Lettered panels) Phalloidin stained embryos. (1) Merged channels of AChR and SV2. (2) Skeletonized images. (3) Magnification of phalloidin channel. (4) Magnification of SV2 channel. (5) Magnification of AChR channel. (6) Magnification of skeleton channel. (A-A6) Control embryo. (B-B6) dag1 morphant embryo. (C-C6) dag1 morphant embryo treated with NAD+ at 6 hpf. (D) Length of skeletons per myotome in control embryos ( $n=186$ half-myotomes), dag 1 morphants ( $n=260$ half-myotomes), and dag1 morphants receiving NAD+ ( $n=252$ half-myotomes) at $6 \mathrm{hpf}$. Skeleton length is not significantly different between untreated and NAD+ treated dagl morphants. (E) Degree of branching within the myotome in control embryos ( $n=180$ halfmyotomes), dag1 morphants ( $n=254$ half-myotomes), and dag1 morphants receiving NAD+ $(n=249$ half-myotomes) at 6 hpf. NAD+ treatment made no significant difference. (F) MTJ angle quantification. MTJ angles are significantly reduced in dag1 morphants receiving NAD+ $(n=98$ MTJs) compared to untreated dag1 morphants ( $n=97$ MTJs). (G) Bar graph of the percent skeleton length of control embryos per myotome. Note that the average percent skeleton length is more drastically reduced in fkrp morphants $(51.4 \%, n=153$ half-myotomes) than in dag 1 morphants (83.9\%, $n=260$ half-myotomes). NAD+ supplementation increased this percentage in fkrp morphants ( $61.9 \%, n=183$ half-myotomes) but has little effect on dag 1 morphants (85.0\%, $n=252$ half-myotomes) Scalebars are $50 \mu \mathrm{m}$, error bars in (G) are standard error of the mean. ${ }^{*} p<0.05,{ }^{* *} p<0.01,{ }^{* * *} p<0.001$, ns non-significant

Organization of laminin at the MTJ is disrupted in zebrafish deficient either for DG [25] or Fkrp [39, 47, 72] (Fig. 1). Laminin is required for concentration of the Integrin adaptor protein Paxillin to the MTJ [24]. Paxillin is an intracellular protein that localizes to cell-ECM adhesion complexes [15] and modulates ECM composition at the developing MTJ [34]. Previous data regarding the beneficial potential of Paxillin in the context of aberrant muscle development/homeostasis are contradictory. Paxillin overexpression worsens muscle damage in ethanol-treated zebrafish [10]. However, Paxillin overexpression is sufficient to improve MTJ morphology in Nrk2b-deficient zebrafish [24]. Paxillin overexpression also improves laminin organization and reduces muscle degeneration in dag1 morphant zebrafish [25]. We hypothesized that Paxillin overexpression would improve muscle structure and function in fkrp morphants where DG is present but not properly glycosylated. Interestingly, we found that Paxillin expression trended towards reducing degeneration and improving the escape response, but the effects were not significant (Fig. 3). This result suggests the hypothesis that, in contrast to the situation where DG is absent, the presence of hypoglycosylated DG in the membrane prevents Paxillin-mediated stabilization of muscle fibers.

\section{Benefits of NAD+ and EmergenC supplementation may be restricted to the neuromusculoskeletal system}

Both fkrp morphants and mutants have an increased UPR. Xpb1, a marker of the UPR, is upregulated in both $f k r p$ morphants and $f k r p$ mutants $[47,63]$. Expression of bip, a marker of the UPR, is upregulated in $f k r p$ morphants at $28 \mathrm{hpf}$, especially in the neural floor plate and the hatching gland [47]. However, glytl1b (large2) morphants with hypoglycosylated DG, dag1 morphants, and sly/lam1c mutants do not exhibit bip upregulation [47], suggesting that activation of the UPR may result directly from loss of Fkrp. We asked if EmergenC supplementation was sufficient to reduce the UPR in $f k r p$ morphants. We found that $3 \mathrm{dpf} T g$ (ef1 $\alpha: x b p 1 \delta$-GFP) embryos injected with $f k r p$ MOs have increased $x b p 1$-GFP fluorescence compared to control embryos. EmergenC supplementation at gastrulation was not sufficient to reduce $x b p 1-G F P$ fluorescence. Taken together, these data suggest that activation of the UPR occurs independently of muscle-cell matrix adhesion and DG glycosylation and is likely a direct consequence of Fkrp knockdown.

Fkrp morphants and fkrp mutants also exhibit aberrant vascular development, including reduced ISV lengths $[63,78]$. One study suggests that impaired vascularization directly results from loss of Fkrp and that muscle phenotypes and vascular phenotypes are independent of one another [78]. Here, we demonstrate that $\mathrm{NAD}+$ supplementation at gastrulation is not sufficient to rescue truncated ISVs in $f k r p$ morphants. Given that $\mathrm{NAD}+$ improves muscle structure and function in $f k r p$ morphants, our data suggest that vascular phenotypes observed in fkrp morphants are not dependent on muscle phenotypes. This supports the previous hypothesis that abnormal vascular development in zebrafish models of FKRP-associated dystroglycanopathy is a direct result of loss of Fkrp.

\section{Timing of NAD+ intervention is critical}

Gene therapy [55, 71, 73, 77], estrogen receptor modulators [79], and exogenous ribitol supplementation [9] improve muscle structure in FKRP-associated dystroglycanopathy models. The efficacy of adenoviral gene therapy and ribitol supplementation decreases if administered later in the mouse's lifespan $[9,77]$, suggesting that early intervention is most beneficial. Our data regarding the timing of NAD+ and EmergenC supplementation in $f k r p$ morphants are consistent with the data mentioned above. We found that $\mathrm{NAD}+$ (or EmergenC) supplementation prior to muscle development increased laminin organization, reduced muscle degeneration, improved muscle function, and improved NMJ structure (Fig. 1). In contrast, NAD+ (or EmergenC) supplementation after initial muscle development only improved MTJs and not muscle structure/function (Fig. 4). 


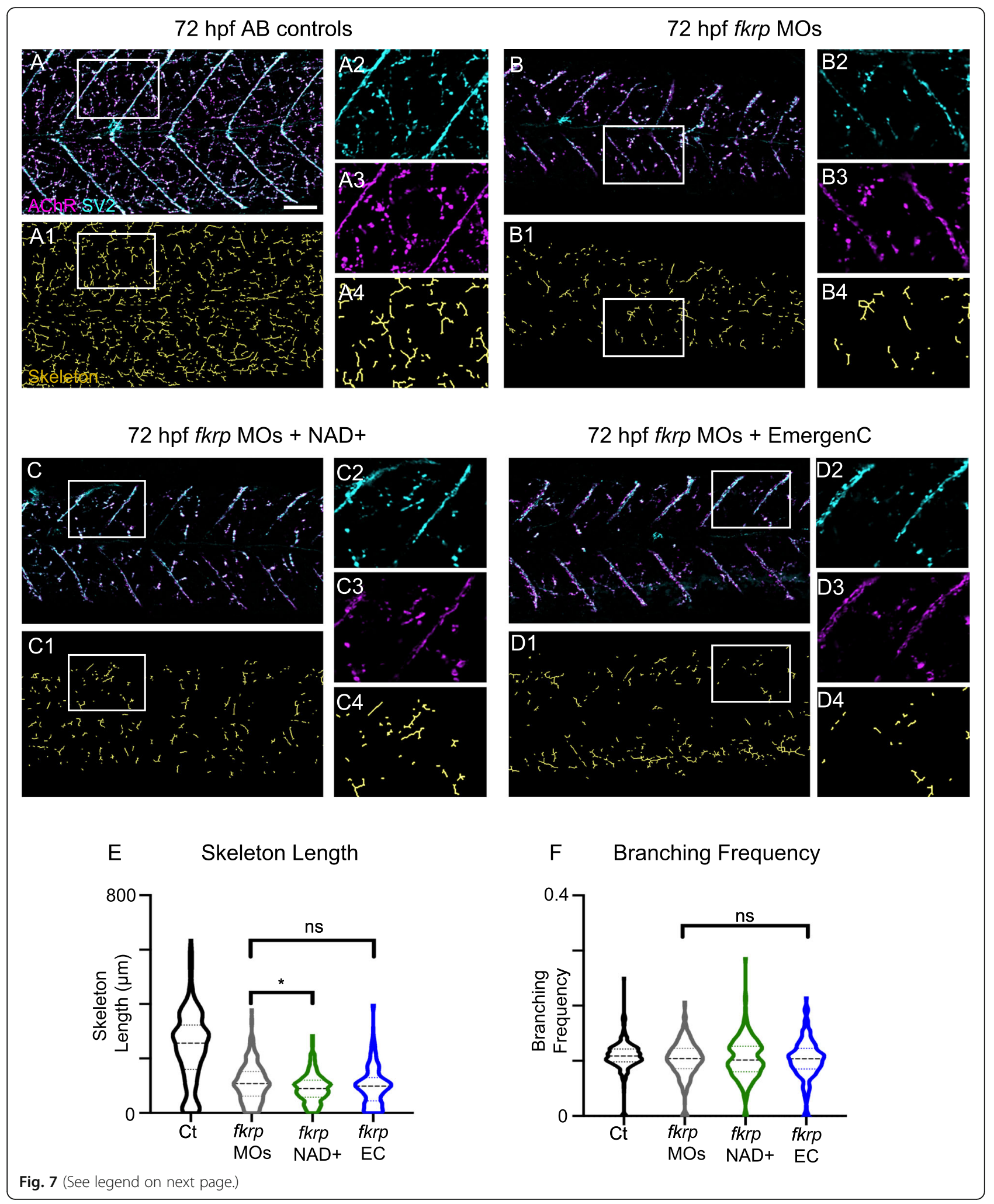


(See figure on previous page.)

Fig. 7 Supplementation with NAD+ or EmergenC after muscle development does not improve NMJ morphology in fkrp morphants. (A-D4) Anterior left, dorsal top, side-mounted embryos at $72 \mathrm{hpf}$ with labeled AChRs and SV2. (Lettered panels) Merged channels of AChR and SV2. (1) Skeletonized images. (2) Magnification of SV2 channel. (3) Magnification of AChR channel. (4) Magnification of skeleton channel. (A-A4) Control embryo. (B-B4) fkrp morphant embryo exhibiting a reduced degree of distributed innervation within the myotome. (C-C4) fkrp morphant embryo treated with NAD+ at $24 \mathrm{hpf}$ also has reduced innervation. (D-D4) fkrp morphant embryo treated with EmergenC at $24 \mathrm{hpf}$ has reduced NMJs. (E) Length of skeletons per myotome in control embryos ( $n=288$ half-myotomes), fkrp morphants ( $n=303$ half-myotomes), and fkrp morphants receiving NAD+ ( $n=225$ half-myotomes) or EmergenC ( $n=225$ half-myotomes) at 24 hpf. Note that skeleton length is actually significantly decreased in fkrp morphants receiving NAD+ at $24 \mathrm{hpf}$. (F) Degree of branching within the myotome in control embryos $(n=283$ half-myotomes), fkrp morphants ( $n=295$ half-myotomes), and fkrp morphants receiving NAD+ $(n=221$ half-myotomes) or EmergenC ( $n=210$ half-myotomes) at $24 \mathrm{hpf}$. Scalebars are $50 \mu \mathrm{m} .{ }^{*} p<0.05,{ }^{* *} p<0.01,{ }^{* * *} p<0.001$, ns non-significant

Our data do not resolve why MTJ morphology is improved with late NAD+ supplementation or muscle-specific overexpression of Fkrp. Improved MTJ morphology does not always correlate with fiber degeneration: itga7 morphants exhibit muscle fiber detachment but have normal MTJ morphology. NAD+ supplementation improves MTJ angles, but not fiber degeneration in dag1/itga7 double morphants [25]. Movement is not necessary for MTJ chevron formation in zebrafish embryos [59]. We show that motility is not improved in $f k r p$ morphants receiving $\mathrm{NAD}+$ or EmergenC after initial muscle development, further suggesting that improved MTJ morphology does not always correlate with mobility.

While this manuscript was in preparation, it was shown that administration of pentetic acid at $48 \mathrm{hpf}$ improved muscle and pericardiac phenotypes in a fkrp mutant zebrafish model of LGMD2I [63]. Pentetic acid is a chelating agent that binds $\mathrm{Ca} 2+$ and $\mathrm{Mg} 2+$. The mechanism by which this improves muscle phenotypes is currently unknown. However, abnormal Ca2+ levels have been implicated in DMD and inducing an influx of $\mathrm{Ca} 2+$ is sufficient to induce dystrophy [50]. Together, these data suggest that different therapeutic avenues have different time windows of efficacy in multiple animal models.

Dag1 and fkrp are required for proper NMJ development NMJ development requires orchestrated interactions between muscle cells and motor neurons. The DGC is a major component of NMJs. Thus, it is not surprising that NMJ morphology is disrupted in multiple dystroglycanopathies. Fukutin-deficient chimeric mice have abnormal AChR clustering and NMJs at postnatal day 15 [61]. Newborn pups homozygous for Large/myd mutations have altered NMJs as well [32]. These data clearly indicate that NMJs are abnormal after birth. What is not clear is at which point in embryonic development NMJ disruption occurs. Do NMJs develop normally and then degenerate or is initial NMJ development abnormal? Here, we provide evidence that early NMJ development is slightly disrupted in DG-deficient zebrafish, with NMJ chains that innervate fast-twitch muscle being approximately $84 \%$ of control chain length. To our knowledge, this is the earliest developmental stage at which NMJ disruption has been observed in a primary or secondary dystroglycanopathy. Interestingly, fkrp morphants exhibit more dramatic disruption of NMJ morphology, with NMJ chains about half of control chain length. We do not know the mechanisms underlying the more severe disruption in NMJ development in fkrp versus dag1 morphants. One possibility is that hypoglycosylated dag1 acts as a "dominant negative" and thus disrupts NMJ development more than when dag1 is not present. Additionally, there are many glycosylated proteins at the NMJ, such as Lrp4, Musk, and Agrin. Thus, the more severe disruption in $f k r p$ morphants could also reflect a requirement of Fkrp for glycosylation of other NMJ resident proteins.

\section{Muscle-specific overexpression of Fkrp is not sufficient to rescue fkrp morphants}

Overexpression of LARGE is sufficient to synthesize glycan-enriched alpha-DG and is sufficient to improve laminin-binding activity in multiple dystroglycanopathy patient cell lines [3]. Muscle-specific expression of Large is sufficient to rescue muscle structure, neurotransmission, and NMJs in Large/myd mutant mice [27]. NMJ defects in Large/myd mutant mice were observed after birth [32]. The above data suggest that, in the context of Large-associated dystroglycanopathy, NMJ defects are secondary to muscle disruption. Given that we observed very early developmental disruption of NMJs, we could not necessarily conclude that NMJ disruption in Fkrp-deficient embryos is secondary to the muscle phenotype. Thus, we tested whether muscle-specific expression of Fkrp would be sufficient to rescue neuromuscular function in $f k r p$ morphant embryos. We first showed that global expression of Fkrp (under control of the heat shock promoter) is sufficient to rescue $f k r p$ morphant embryos. This result indicates that the Fkrp-EGFP fusion protein is functional.

Next, we expressed Fkrp in muscle under control of the muscle specific -503unc promoter [5]. Muscle-specific expression of Fkrp was sufficient to slightly, but significantly, reduce MTJ angles. However, muscle-specific expression of Fkrp was not sufficient to reduce muscle degeneration or improve the escape response (Fig. 8). We do not know 


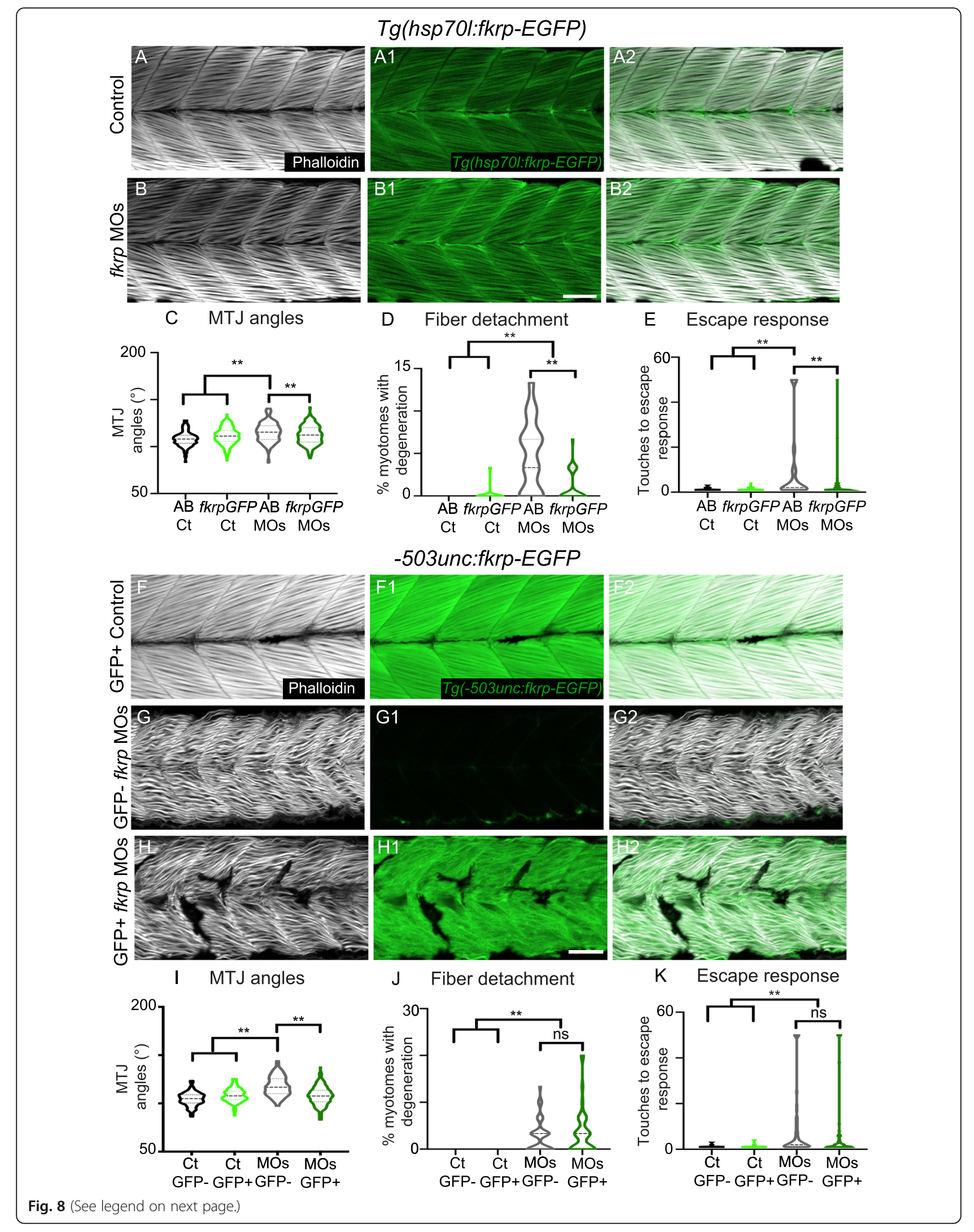


(See figure on previous page.)

Fig. 8 Muscle-specific overexpression of Fkrp improves MTJ angles, but not motility or fiber resiliency. (A-B2) Anterior left, dorsal top, sidemounted embryos at $72 \mathrm{hpf}$ stained for f-actin (phalloidin, gray) and expressing Fkrp-EGFP (green). (A-A2) Control Tg (hsp70l:fkrp-EGFP) embryo expressing Fkrp in the fibers. (B-B2) fkrp morphant Tg (hsp70l:fkrp-EGFP) embryo expressing Fkrp in fibers. (C) MTJ angles of Tg (hsp70l:fkrp-EGFP) control $(n=264$ MTJs) and fkrp morphant ( $n=340$ MTJs) embryos and AB controls and morphants $(n=141,194$ MTJs) at 72 hpf. Constitutive expression of Fkrp improves MTJ angles in morphants. (D) Constitutive expression of Fkrp in morphants ( $n=72$ embryos) significantly reduces the number of myotomes with fiber degeneration compared with control morphants ( $n=35$ embryos). (E) The escape response is significantly reduced in fkrp morphants constitutively overexpressing Fkrp ( $n=82$ embryos) compared to control morphants $(n=44$ embryos). (F-H2) Anterior left, dorsal top, side-mounted embryos at $72 \mathrm{hpf}$ stained for f-actin (phalloidin, gray) and expressing Fkrp-EGFP under control of the musclespecific -503unc promoter (green). (F) Control Tg(-503unc:fkrp-EGFP) embryo expressing Fkrp specifically in muscle fibers. G) fkrp morphant embryo on $T g(-503 u n c: f k r p-E G F P)$ background lacking Fkrp expression in muscle fibers. (H) fkrp morphant Tg(-503unc:fkrp-EGFP) embryo expressing Fkrp specifically in muscle fibers does not show improved muscle organization. (I) Muscle specific expression of FKRP in fkrp morphants ( $n=158$ MTJs) significantly improves MTJ angles compared to control morphants ( $n=153 \mathrm{MTJs}$ ). (J) Muscle-specific overexpression of Fkrp ( $n=45$ embryos) does not significantly lower the percent of myotomes with muscle degeneration in control fkrp morphants $(n=47$ embryos). (K) There is not a significant difference in the number of touches required to induce an escape response in $f k r p$ morphants that overexpress Fkrp ( $n=73$ embryos) in muscle versus those that do not ( $n=79$ embryos). Scalebars are $50 \mu \mathrm{m} .{ }^{*} p<0.05$, ${ }^{* *} p<0.01$, ns non-significant

why our results differ from the Large study. We do not believe that the timing of expression is the key factor because muscle creatine kinase (Large study) and Unc45b (our study) are expressed at similar times during myotome development. In the DG glycosylation pathway, FKRP works in concert with fukutin (FKTN) to synthesize a tandem ribitol phosphate that connects a core o-linked glycan with matriglycan, the ligand for laminin and other ECM proteins [38]. This step precedes the completion of matriglycan synthesis by LARGE. Roles for LARGE or FKRP in other glycosylation reactions have not been identified. However, the fact that muscle-specific expression of Fkrp is not sufficient to rescue muscle homeostasis may suggest that Fkrp is required to glycosylate proteins other than DG. Regardless, our data indicate that Fkrp is required outside of skeletal muscle to reduce muscle degeneration. In the future, it will be interesting to determine in which tissues and cells Fkrp is required to rescue neuromuscular function in Fkrp-deficient embryos.

\section{Conclusion}

The myomatrix is critical for muscle development and homeostasis and is disrupted in many dystroglycanopathies studied thus far $[17,47,48,58,68]$. Here, we primarily focused on two specialized junctions within the myomatrix: the MTJ and NMJ. MTJ and NMJ development are disrupted in $f k r p$ morphants. NAD+ supplementation prior to muscle development, which improves myomatrix organization in DG-deficient zebrafish, was sufficient to improve muscle homeostasis, MTJ structure, and NMJ formation in fkrp morphant zebrafish. However, NAD+ did not improve vascular or UPR phenotypes in fkrp morphants, and NAD+ supplementation after initial muscle development was not particularly efficacious. There was a slight improvement in MTJ structure and a reduced variation in muscle degeneration. These data are similar to previous studies showing that earlier intervention has improved outcomes in FKRP mutant mice [77]. Interestingly, in contrast to muscle- specific expression of Large in Large/myd mutant mice, muscle-specific overexpression of Fkrp was not sufficient to improve muscle structure/function in $f k r p$ morphants. Taken together, our data indicate that Fkrp plays an early and crucial role in muscle, MTJ, and NMJ development.

\section{Additional file}

Additional file 1 : Figure S1 Scoring of relative MTJ staining intensity. (A-C) Anterior left, dorsal top, side-mounted zebrafish embryos at $26 \mathrm{hpf}$ stained for laminin-111 at the MTJ (purple). (A) Example of an embryo with strong laminin staining intensity at the MTJ. (B) An embryo with weak laminin staining intensity at the MTJ. (C) An embryo lacking laminin staining at the MTJ. (EPS $1671 \mathrm{~kb}$ )

\section{Abbreviations}

AChR: Acetylcholine receptor; DG: Dystroglycan; DGC: Dystrophinglycoprotein complex; DMD: Duchenne Muscular Dystrophy; dpf: Days postfertilization; ECM: Extracellular matrix; ER: Endoplasmic reticulum; ERM: Embryo rearing medium; FKRP: Fukutin-related protein; GFP: Green fluorescent protein; GMPPB: GDP-mannose Pyrophosphorylase B; hpf: Hours post-fertilization; ISPD: Isoprenoid Synthase Domain-Containing; ISV: Intersegmental vessel; Itga7: Integrin alpha7; LGMD2I: Limb-Girdle Muscular Dystrophy Type 2l; MO: Morpholino; MTJ: Myotendinous junction; NMJ: Neuromuscular junction; NAD+: Nicotinamide riboside; Nrk2b: Nicotinamide riboside kinase 2b; PFA: Paraformaldehyde; SV2: Synaptic vesicle protein 2; Tg: Transgenic; UPR: Unfolded protein response

\section{Acknowledgements}

The authors would like to thank the Henry lab, especially Dr. Michelle Goody for technical assistance and critical reading of the manuscript, Mary Astumian for technical assistance and generation of transgenic fish, Amneh Wise and Ethan King for deyolking assistance, and Maggie Pasquarella for her assistance with preliminary experiments. We also thank Wyatt Austin for insight regarding image analysis and Dr. Benjamin King and Dexter Canning for insight on statistics. We also thank Mark Nilan for outstanding fish care and maintenance.

\section{Authors' contributions}

The authors have made the following declarations about their contributions: ECB and $\mathrm{CAH}$ conceived and designed experiments, performed experiments, analyzed the data, and prepared the manuscript. SSA, ESC, and DMD performed experiments and analyzed the data. JJB and CMK performed experiments. EAK analyzed the data and contributed analysis tools. AK contributed analysis tools. JBK analyzed the data, developed analysis tools, and prepared the manuscript. All authors read and approved the final manuscript. 


\section{Funding}

This work was supported by the March of Dimes Award number \#1-FY14-284 to C.A.H., the National Institute of Child Health and Human Development Award number R15HD088217 to C.A.H., and the University of Maine Chase Distinguished Research Assistantship to E.C.B. The funders had no role in study design, data collection, data analysis, decision to publish, or preparation of the manuscript.

\section{Availability of data and materials}

All detailed statistical analyses and a compilation of all images will be uploaded to figshare upon acceptance.

\section{Consent for publication}

Not applicable

\section{Competing interests}

The authors declare that they have no competing interests.

\section{Author details}

'School of Biology and Ecology, University of Maine, Orono, ME 04469, USA ${ }^{2}$ Chemical and Biomedical Engineering, University of Maine, Orono, ME 04469, USA. ${ }^{3}$ Molecular and Biomedical Sciences, University of Maine, Orono, ME 04469, USA. ${ }^{4}$ Graduate School of Biomedical Sciences and Engineering, University of Maine, 217 Hitchner Hall, Orono, ME 04469, USA. ${ }^{5}$ Present Address: Tufts University School of Medicine, Boston, MA 02111, USA. ${ }^{6}$ Present Address: Lake Erie College of Osteopathic Medicine, Erie, PA 16509, USA.

Received: 25 March 2019 Accepted: 17 July 2019 Published online: 07 August 2019

\section{References}

1. Ackroyd MR, Skordis L, Kaluarachchi M, Godwin J, Prior S, Fidanboylu M, Piercy RJ, Muntoni F, Brown SC. Reduced expression of fukutin related protein in mice results in a model for fukutin related protein associated muscular dystrophies. Brain J Neurol. 2009;132(Pt 2):439-51 https://doi.org/1 0.1093/brain/awn335.

2. Bajanca F, Luz M, Raymond K, Martins GG, Sonnenberg A, Tajbakhsh S, Buckingham M, Thorsteinsdóttir S. Integrin Alpha6beta1-laminin interactions regulate early myotome formation in the mouse embryo. Development (Cambridge, England). 2006;133(9):1635-44. https://doi.org/10.1242/dev.02336.

3. Barresi R, Michele DE, Kanagawa M, Harper HA, Dovico SA, Satz JS, Moore $S A$, et al. LARGE can functionally bypass alpha-dystroglycan glycosylation defects in distinct congenital muscular dystrophies. Nat Med. 2004;10(7): 696-703. https://doi.org/10.1038/nm1059.

4. Beltran-Valero de Bernabé D, Voit T, Longman C, Steinbrecher A, Straub $V$, Yuva Y, Herrmann R, et al. Mutations in the FKRP gene can cause muscle-eye-brain disease and Walker-Warburg syndrome. J Med Genet. 2004;41(5):e61 https://doi.org/10.1136/jmg.2003.013870.

5. Berger J, Currie PD. 503unc, a small and muscle-specific zebrafish promoter. Genesis (New York, N.Y.: 2000). 2013;51(6):443-7 https://doi. org/10.1002/dvg.22385.

6. Bernabé $D$, Beltrán-Valero de $H$, van Bokhoven $E$, van Beusekom W, Van den Akker S, Kant W, B Dobyns, B Cormand, et al. "A homozygous nonsense mutation in the fukutin gene causes a Walker-Warburg syndrome phenotype." J Med Genet 200340 (11): 845-848. https://doi. org/10.1136/jmg.40.11.845

7. Bieganowski $P$, Brenner $C$. Discoveries of nicotinamide riboside as a nutrient and conserved NRK genes establish a Preiss-Handler independent route to NAD+ in Fungi and humans. Cell. 2004;117(4):495-502.

8. Briggs DC, Yoshida-Moriguchi T, Zheng T, Venzke D, Anderson ME, Strazzulli A, Moracci M, Yu L, Hohenester E, Campbell KP. Structural basis of laminin binding to the LARGE glycans on dystroglycan. Nat Chem Biol. 2016;12(10): 810-4 https://doi.org/10.1038/nchembio.2146.

9. Cataldi MP, Lu P, Blaeser A, Qi Long L. Ribitol restores functionally glycosylated a-Dystroglycan and improves muscle function in dystrophic FKRP-mutant mice. Nat Commun. 2018;9(1):3448 https://doi.org/10.1038/ s41467-018-05990-z.

10. Coffey EC, Pasquarella ME, Goody MF, Henry CA. Ethanol exposure causes muscle degeneration in zebrafish. J Dev Biol. 2018;6(1) https:// doi.org/10.3390/jdb6010007.
11. Cohn RD, Mayer U, Saher G, Herrmann R, van der Flier A, Sonnenberg A, Sorokin L, Voit T. Secondary reduction of Alpha7B integrin in laminin Alpha2 deficient congenital muscular dystrophy supports an additional transmembrane link in skeletal muscle. J Neurol Sci. 1999;163(2):140-52.

12. Constantin B. Dystrophin complex functions as a scaffold for signalling proteins. Biochim Biophys Acta. 2014;1838(2):635-42 https://doi.org/10.1016/ j.bbamem.2013.08.023.

13. Côté PD, Moukhles H, Lindenbaum M, Carbonetto S. Chimaeric mice deficient in dystroglycans develop muscular dystrophy and have disrupted Myoneural synapses. Nat Genet. 1999;23(3):338-42 https://doi.org/10.1038/15519.

14. Crawford BD, Henry CA, Clason TA, Becker AL, Hille MB. Activity and distribution of paxillin, focal adhesion kinase, and cadherin indicate cooperative roles during zebrafish morphogenesis. Mol Biol Cell. 2003;14(8): 3065-81 https://doi.org/10.1091/mbc.e02-08-0537.

15. Deakin NO, Turner CE. Paxillin comes of age. J Cell Sci. 2008;121(Pt 15): 2435-44 https://doi.org/10.1242/jcs.018044.

16. Deconinck AE, Potter AC, Tinsley JM, Wood SJ, Vater R, Young C, Metzinger L, Vincent A, Slater CR, Davies KE. Postsynaptic abnormalities at the neuromuscular junctions of utrophin-deficient mice. J Cell Biol. 1997;136(4): 883-94 https://doi.org/10.1083/jcb.136.4.883

17. Di Costanzo S, Balasubramanian A, Pond HL, Rozkalne A, Pantaleoni C, Saredi S, Gupta VA, et al. POMK mutations disrupt muscle development leading to a spectrum of neuromuscular presentations. Hum Mol Genet. 2014;23(21):5781-92 https://doi.org/10.1093/hmg/ddu296.

18. Ervasti JM, Campbell KP. A role for the dystrophin-glycoprotein complex as a transmembrane linker between laminin and actin. J Cell Biol. 1993;122(4): 809-23 https://doi.org/10.1083/jcb.122.4.809.

19. Gawlik K, Miyagoe-Suzuki Y, Ekblom P, Takeda S'i, Durbeej M. Laminin Alpha1 chain reduces muscular dystrophy in laminin Alpha2 chain deficient mice. Hum Mol Genet. 2004;13(16):1775-84 https://doi.org/10.1093/hmg/ddh190.

20. Gee SH, Montanaro F, Lindenbaum MH, Carbonetto S. Dystroglycanalpha, a dystrophin-associated glycoprotein, is a functional Agrin receptor. Cell. 1994;77(5):675-86.

21. Gerin I, Ury B, Breloy I, Bouchet-Seraphin C, Bolsée J, Halbout M, Graff J, et al. ISPD produces CDP-ribitol used by FKTN and FKRP to transfer ribitol phosphate onto a-dystroglycan. Nat Commun. 2016;7:11534 https://doi. org/10.1038/ncomms11534.

22. Goddeeris MM, Wu B, Venzke D, Yoshida-Moriguchi T, Saito F, Matsumura K, Moore SA, Campbell KP. LARGE Glycans on dystroglycan function as a tunable matrix scaffold to prevent dystrophy. Nature. 2013;503(7474):136-40 https://doi.org/10.1038/nature12605.

23. Godfrey C, Reghan Foley A, Clement E, Muntoni F. Dystroglycanopathies: coming into focus. Curr Opin Genet Dev. 2011;21(3):278-85 https://doi.org/1 0.1016/j.gde.2011.02.001.

24. Goody MF, Kelly MW, Lessard KN, Khalil A, Henry CA. Nrk2b-mediated NAD+ production regulates cell adhesion and is required for muscle morphogenesis in vivo: Nrk2b and NAD+ in muscle morphogenesis. Dev Biol. 2010;344(2):809-26 https://doi.org/10.1016/j.ydbio.2010.05.513.

25. Goody MF, Kelly MW, Reynolds CJ, Khalil A, Crawford BD, Henry CA. NAD+ biosynthesis ameliorates a zebrafish model of muscular dystrophy. PLoS Biol. 2012;10(10):e1001409 https://doi.org/10.1371/journal.pbio.1001409.

26. Goody MF, Sher RB, Henry CA. Hanging on for the ride: adhesion to the extracellular matrix mediates cellular responses in skeletal muscle morphogenesis and disease. Dev Biol. 2015;401(1):75-91 https://doi.org/10.1 016/j.ydbio.2015.01.002.

27. Gumerson JD, Davis CS, Kabaeva ZT, Hayes JM, Brooks SV, Michele DE. Muscle-specific expression of LARGE restores neuromuscular transmission deficits in dystrophic LARGE (Myd) mice. Hum Mol Genet. 2013;22(4):757-68 https://doi.org/10.1093/hmg/dds483.

28. Gupta VA, Kawahara G, Myers JA, Chen AT, Hall TE, Chiara Manzini M, Currie $P D$, et al. A splice site mutation in laminin-A2 results in a severe muscular dystrophy and growth abnormalities in zebrafish. PLoS One. 2012;7(8): e43794 https://doi.org/10.1371/journal.pone.0043794.

29. Hall TE, Bryson-Richardson RJ, Berger S, Jacoby AS, Cole NJ, Hollway GE, Berger J, Currie PD. The zebrafish candyfloss mutant implicates extracellular matrix adhesion failure in laminin Alpha2-deficient congenital muscular dystrophy. Proc Natl Acad Sci U S A. 2007;104(17):7092-7 https://doi.org/1 0.1073/pnas.0700942104.

30. Hayashi YK, Chou FL, Engvall E, Ogawa M, Matsuda C, Hirabayashi S, Yokochi $\mathrm{K}$, et al. Mutations in the integrin Alpha7 gene cause congenital myopathy. Nat Genet. 1998;19(1):94-7 https://doi.org/10.1038/ng0598-94. 
31. Helbling-Leclerc A, Zhang X, Topaloglu H, Cruaud C, Tesson F, Weissenbach J, Tomé FM, Schwartz K, Fardeau M, Tryggvason K. Mutations in the laminin alpha 2-chain gene (LAMA2) cause merosin-deficient congenital muscular dystrophy. Nat Genet. 1995;11(2):216-8 https://doi.org/10.1038/ng1095-216.

32. Herbst $R$, Iskratsch $T$, Unger $E$, Bittner RE. Aberrant development of neuromuscular junctions in glycosylation-defective Large (Myd) mice. Neuromuscular Disorders: NMD. 2009;19(5):366-78 https://doi.org/10.1016/j. nmd.2009.02.011.

33. Inamori K-i, Yoshida-Moriguchi T, Hara Y, Anderson ME, Yu L, Campbell KP. Dystroglycan function requires xylosyl- and glucuronyltransferase activities of LARGE. Science (New York, N.Y.). 2012;335(6064):93-6 https://doi.org/1 $0.1126 /$ science. 1214115 .

34. Jacob AE, Amack JD, Turner CE. Paxillin genes and actomyosin contractility regulate myotome morphogenesis in zebrafish. Dev Biol. 2017:425(1):70-84 https://doi.org/10.1016/j.ydbio.2017.03.012

35. Jayasinha V, Nguyen HH, Xia B, Kammesheidt A, Hoyte K, Martin PT. Inhibition of dystroglycan cleavage causes muscular dystrophy in transgenic mice. Neuromuscul Disord. 2003;13(5):365-75.

36. Jensen, Braden S., Tobias Willer, Dimah N. Saade, Mary O. Cox, Tahseen Mozaffar, Mena Scavina, Vikki A. Stefans, et al. "GMPPB-associated dystroglycanopathy: emerging common variants with phenotype correlation." Hum Mutat 201536 (12): 1159-1163. https://doi.org/10.1 002/humu.22898

37. Johnson K, Bertoli M, Phillips L, Töpf A, Van den Bergh P, Vissing J, Witting $\mathrm{N}$, et al. Detection of variants in dystroglycanopathy-associated genes through the application of targeted whole-exome sequencing analysis to a Large cohort of patients with unexplained limb-girdle muscle weakness. Skelet Muscle. 2018;8(1):23 https://doi.org/10.1186/s13395-018-0170-1.

38. Kanagawa M, Kobayashi K, Tajiri M, Manya H, Kuga A, Yamaguchi Y, Akasaka-Manya $\mathrm{K}$, et al. Identification of a post-translational modification with ribitol-phosphate and its defect in muscular dystrophy. Cell Rep. 2016; 14(9):2209-23 https://doi.org/10.1016/j.celrep.2016.02.017.

39. Kawahara G, Guyon JR, Nakamura Y, Kunkel LM. Zebrafish models for human FKRP muscular dystrophies. Hum Mol Genet. 2010;19(4):623-33 https://doi.org/10.1093/hmg/ddp528.

40. Kimmel CB, Ballard WW, Kimmel SR, Ullmann B, Schilling TF. Stages of embryonic development of the zebrafish. Dev Dyn. 1995;203(3):253-310 https://doi.org/10.1002/aja.1002030302.

41. Kwan KM, Fujimoto E, Grabher C, Mangum BD, Hardy ME, Campbell DS, Parant JM, Joseph Yost H, Kanki JP, Chien C-B. The Tol2kit: a multisite gateway-based construction kit for Tol2 transposon Transgenesis constructs. Dev Dyn. 2007;236(11):3088-99 https://doi.org/10.1002/dvdy.21343.

42. Lawson ND, Weinstein BM. In vivo imaging of embryonic vascular development using transgenic zebrafish. Dev Biol. 2002;248(2):307-18.

43. Levedakou EN, Chen X-J, Soliven B, Popko B. Disruption of the mouse Large gene in the Enr and Myd mutants results in nerve, muscle, and neuromuscular junction defects. Mol Cell Neurosci. 2005;28(4):757-69 https://doi.org/10.1016/j.mcn.2004.12.007.

44. Li J, Mayne R, Wu C. A novel muscle-specific Beta 1 integrin binding protein (MIBP) that modulates myogenic differentiation. J Cell Biol. 1999;147(7): 1391-8 https://doi.org/10.1083/jcb.147.7.1391.

45. Li J, Rao H, Burkin D, Kaufman SJ, Chuanyue W. The muscle integrin binding protein (MIBP) interacts with Alpha7beta1 integrin and regulates cell adhesion and laminin matrix deposition. Dev Biol. 2003;261(1):209-19.

46. Li J, Chen Z, Gao L-Y, Colorni A, Ucko M, Fang S, Shao Jun D. A transgenic zebrafish model for monitoring Xbp1 splicing and endoplasmic reticulum stress in vivo. Mech Dev. 2015;137(August):33-44 https://doi.org/10.1016/j. mod.2015.04.001.

47. Lin Y-Y, White RJ, Torelli S, Cirak S, Muntoni F, Stemple DL. Zebrafish Fukutin family proteins link the unfolded protein response with dystroglycanopathies. Hum Mol Genet. 2011;20(9):1763-75 https://doi.org/1 0.1093/hmg/ddr059.

48. Marchese M, Pappalardo A, Baldacci J, Verri T, Doccini S, Cassandrini D, Bruno C, et al. Dolichol-phosphate mannose synthase depletion in zebrafish leads to dystrophic muscle with hypoglycosylated a-dystroglycan. Biochem Biophys Res Commun. 2016:477(1):137-43 https://doi.org/10.1016/j.bbrc.2016.06.033.

49. Martin PT. Mechanisms of Disease: Congenital Muscular DystrophiesGlycosylation Takes Center Stage. Nat Clin Pract Neurol. 2006;2(4):222-30 https://doi.org/10.1038/ncpneuro0155.

50. Millay DP, Goonasekera SA, Sargent MA, Maillet M, Aronow BJ, Molkentin JD. Calcium influx is sufficient to induce muscular dystrophy through a TRPC-dependent mechanism. Proc Natl Acad Sci U S A. 2009; 106(45):19023-8 https://doi.org/10.1073/pnas.0906591106.

51. Muntoni F, Torelli S, Wells DJ, Brown SC. Muscular dystrophies due to glycosylation defects: diagnosis and therapeutic strategies. Curr Opin Neurol. 2011;24(5):437-42 https://doi.org/10.1097/WCO.0b013e32834a95e3.

52. Nickolls AR, Bönnemann CG. The roles of dystroglycan in the nervous system: insights from animal models of muscular dystrophy. Dis Model Mech. 2018;11(12) https://doi.org/10.1242/dmm.035931.

53. Parsons MJ, Campos I, Elizabeth M, Hirst A, Stemple DL. Removal of dystroglycan causes severe muscular dystrophy in zebrafish embryos. Development (Cambridge, England). 2002;129(14):3505-12.

54. Pratt SJP, Valencia AP, Le GK, Shah SB, Lovering RM. Pre- and postsynaptic changes in the neuromuscular junction in dystrophic mice. Front Physiol. 2015;6:252 https://doi.org/10.3389/fphys.2015.00252.

55. Qiao C, Wang C-H, Zhao C, Lu P, Awano H, Xiao B, Li J, et al. Muscle and heart function restoration in a limb girdle muscular dystrophy 2 (LGMD2l) mouse model by systemic FKRP gene delivery. Mol Ther. 2014;22(11):1890-9 https://doi.org/10.1038/mt.2014.141.

56. Rooney JE, Gurpur PB, Burkin DJ. Laminin-111 protein therapy prevents muscle disease in the mdx mouse model for Duchenne muscular dystrophy. Proc Natl Acad Sci U S A. 2009;106(19):7991-6 https://doi.org/1 0.1073/pnas.0811599106

57. Rooney JE, Knapp JR, Hodges BL, Wuebbles RD, Burkin DJ. Laminin-111 protein therapy reduces muscle pathology and improves viability of a mouse model of Merosin-deficient congenital muscular dystrophy. Am J Pathol. 2012;180(4):1593-602 https://doi.org/10.1016/j.ajpath.2011.12.019.

58. Roscioli T, Kamsteeg E-J, Buysse K, Maystadt I, van Reeuwijk J, van den Elzen C, van Beusekom E, et al. Mutations in ISPD cause Walker-Warburg syndrome and defective glycosylation of a-dystroglycan. Nat Genet. 2012; 44(5):581-5 https://doi.org/10.1038/ng.2253.

59. Rost F, Eugster C, Schröter C, Oates AC, Brusch L. Chevron formation of the zebrafish muscle segments. J Exp Biol. 2014;217(Pt 21):3870-82 https://doi. org/10.1242/jeb.102202.

60. Rybakova IN, Patel JR, Ervasti JM. The dystrophin complex forms a mechanically strong link between the sarcolemma and costameric actin. J Cell Biol. 2000;150(5):1209-14 https://doi.org/10.1083/jcb.150.5.1209.

61. Saito F, Masaki T, Saito Y, Nakamura A, Takeda S, Shimizu T, Toda T, Matsumura K. Defective peripheral nerve myelination and neuromuscular junction formation in fukutin-deficient chimeric mice. J Neurochem. 2007; 101(6):1712-22 https://doi.org/10.1111/j.1471-4159.2007.04462.x

62. Sarathy A, Wuebbles RD, Fontelonga TM, Tarchione AR, Mathews Griner LA, Heredia DJ, Nunes AM, et al. SU9516 increases A7ß1 integrin and ameliorates disease progression in the mdx mouse model of duchenne muscular dystrophy. Mol Ther. 2017;25(6):1395-407 https://doi.org/10.1016/j. ymthe.2017.03.022.

63. Serafini PR, Feyder MJ, Hightower RM, Garcia-Perez D, Vieira NM, Lek A, Gibbs DE, et al. A limb-girdle muscular dystrophy 21 model of muscular dystrophy identifies corrective drug compounds for dystroglycanopathies. JCI Insight. 2018;3(18) https://doi.org/10.1172/jci.insight.120493.

64. Smith SJ, Wang JC, Gupta VA, Dowling JJ. A novel early onset phenotype in a zebrafish model of merosin deficient congenital muscular dystrophy. PLoS One. 2017;12(2):e0172648 https://doi.org/10.1371/journal.pone.0172648.

65. Snow CJ, Goody M, Kelly MW, Oster EC, Jones R, Khalil A, Henry CA. Time-lapse analysis and mathematical characterization elucidate novel mechanisms underlying muscle morphogenesis. PLoS Genet. 2008;4(10):e1000219 https:// doi.org/10.1371/journal.pgen.1000219.

66. Song WK, Wang W, Sato H, Bielser DA, Kaufman SJ. Expression of alpha 7 integrin cytoplasmic domains during skeletal muscle development: alternate forms, conformational change, and homologies with serine/threonine kinases and tyrosine phosphatases. J Cell Sci. 1993;106(Pt 4):1139-52.

67. Stensland E, Lindal S, Jonsrud C, Torbergsen T, Bindoff LA, Rasmussen M, Dahl A, Thyssen F, Nilssen $\varnothing$. Prevalence, mutation spectrum and phenotypic variability in Norwegian patients with limb girdle muscular dystrophy 2l. Neuromuscul Disord. 2011;21(1):41-6 https://doi.org/10.1016/j. nmd.2010.08.008.

68. Stevens E, Carss KJ, Sebahattin C, Reghan Foley A, Torelli S, Willer T, Tambunan DE, et al. Mutations in B3GALNT2 cause congenital muscular dystrophy and Hypoglycosylation of a-dystroglycan. Am J Hum Genet. 2013;92(3):354-65 https://doi.org/10.1016/j.ajhg.2013.01.016.

69. Taniguchi-Ikeda M, Morioka I, lijima K, Toda T. Mechanistic aspects of the formation of a-Dystroglycan and therapeutic research for the treatment of 
a-Dystroglycanopathy: A review. Mol Asp Med. 2016;51:115-24 https://doi. org/10.1016/j.mam.2016.07.003.

70. Tempel W, Rabeh WM, Bogan KL, Belenky P, Wojcik M, Seidle HF, Nedyalkova $L$, et al. Nicotinamide riboside kinase structures reveal new pathways to NAD+. PLoS Biol. 2007;5(10):e263 https://doi.org/10.1371/ journal.pbio.0050263.

71. Thomas PJ, Xu R, Martin PT. B4GALNT2 (GALGT2) gene therapy reduces skeletal muscle pathology in the FKRP P448L mouse model of limb girdle muscular dystrophy 21. Am J Pathol. 2016;186(9):2429-48 https://doi.org/10.1 016/j.ajpath.2016.05.021.

72. Thornhill P, Bassett D, Lochmüller H, Bushby K, Straub V. Developmental defects in a zebrafish model for muscular dystrophies associated with the loss of fukutin-related protein (FKRP). Brain J Neurol. 2008;131(Pt 6):1551-61 https://doi.org/10.1093/brain/awn078.

73. Tucker JD, Lu PJ, Xiao X, Qi LL. Overexpression of mutant FKRP restores functional glycosylation and improves dystrophic phenotype in FKRP mutant mice. Mol Ther Nucleic Acids. 2018;11(June):216-27 https://doi.org/1 0.1016/j.omtn.2018.02.008.

74. Turner CE, Glenney JR, Burridge K. Paxillin: A new vinculin-binding protein present in focal adhesions. J Cell Biol. 1990;111(3):1059-68 https://doi.org/1 0.1083/jcb.111.3.1059.

75. Van Reeuwijk J, Olderode-Berends MJW, Van den Elzen C, Brouwer OF, Roscioli T, Van Pampus MG, Scheffer H, Brunner HG, Van Bokhoven H, Hol FA. A homozygous FKRP start codon mutation is associated with Walker-Warburg syndrome, the severe end of the clinical spectrum. Clin Genet. 2010;78(3):275-81 https://doi.org/10.1111/j.1399-0004.2010.01384.X

76. Ry V, Pam M, Wuebbles RD, Key M, Burkin DJ. Galectin-1 protein therapy prevents pathology and improves muscle function in the $\mathrm{mdx}$ mouse model of Duchenne muscular dystrophy. Mol Ther. 2015;23(8):1285-97 https://doi.org/10.1038/mt.2015.105.

77. Vannoy CH, Xiao W, Lu P, Xiao X, Qi Long L. Efficacy of gene therapy is dependent on disease progression in dystrophic mice with mutations in the FKRP gene. Mol Ther Methods Clin Dev. 2017:5(June):31-42 https://doi. org/10.1016/j.omtm.2017.02.002.

78. Wood AJ, Müller JS, Jepson CD, Laval SH, Lochmüller H, Bushby K, Barresi R, Straub V. Abnormal vascular development in zebrafish models for fukutin and FKRP deficiency. Hum Mol Genet. 2011;20(24):4879-90 https://doi.org/1 0.1093/hmg/ddr426.

79. Wu B, Shah SN, Lu P, Bollinger LE, Blaeser A, Sparks S, Harper AD, Qi LL. Longterm treatment of tamoxifen and Raloxifene alleviates dystrophic phenotype and enhances muscle functions of FKRP gystroglycanopathy. Am J Pathol. 2018;188(4):1069-80 https://doi.org/10.1016/j.ajpath.2017.12.011.

80. Yurchenco PD, McKee KK, Reinhard JR, Rüegg MA. Laminin-deficient muscular dystrophy: molecular pathogenesis and structural repair strategies. Matrix Biol. 2018;71-72:174-87 https://doi.org/10.1016/j.matbio.2017.11.009.

\section{Publisher's Note}

Springer Nature remains neutral with regard to jurisdictional claims in published maps and institutional affiliations.

Ready to submit your research? Choose BMC and benefit from:

- fast, convenient online submission

- thorough peer review by experienced researchers in your field

- rapid publication on acceptance

- support for research data, including large and complex data types

- gold Open Access which fosters wider collaboration and increased citations

- maximum visibility for your research: over $100 \mathrm{M}$ website views per year

At BMC, research is always in progress.

Learn more biomedcentral.com/submissions 